\title{
How topography-dependent are topographic effects? Complementary numerical modeling of centrifuge experiments
}

\author{
Seokho Jeong a,*, Domniki Asimaki ${ }^{\mathrm{b}}$, Jacob Dafni ${ }^{\mathrm{c}}$, Joseph Wartman ${ }^{\mathrm{c}}$ \\ ${ }^{a}$ School of Engineering, University of Waikato, Hamilton 3240, New Zealand \\ ${ }^{b}$ Department of Mechanical and Civil Engineering, California Institute of Technology, Pasadena, CA 91125, USA \\ ${ }^{c}$ Department of Civil and Environmental Engineering, University of Washington, Seattle, WA 98195, USA
}

\begin{abstract}
Topographic effects, the modification of seismic shaking by irregular topographies compared to flat ground, have been extensively studied. Very few studies, however, have investigated the effects of the stratigraphy and nonlinear response of the underlying geology on topographic amplification. Furthermore, most experimental studies have been performed in the field, where it is often difficult to establish an ideal flat-ground reference station, as well as to characterize the soil properties and their spatial variability in sufficient detail. Dafni [1] recently tested the seismic response of step-like slopes in a series of centrifuge experiments, where the incident motion, reference station and material properties were characterized in detail. In this study, we investigated the influence of the container boundary on topographic effects observed in the centrifuge experiments by performing numerical simulations with and without the container boundary. Our analysis suggested that the rigid-body rocking motion of the centrifuge container likely increased the experimental topographic spectral ratios, contributing to the discrepancy between the simulated and observed spectral ratios. We also found that although the laminar box lateral boundaries caused spurious reflections, they didn't qualitatively affect the ground surface amplification pattern compared to numerical predictions of the same configuration without boundaries. At the same time, and most importantly, however, we found that the baseplate -by trapping waves scattered and diffracted by the slopeamplified the ground motion at the crest up to one order of magnitude compared to numerical predictions of the response in absence of the baseplate. Our results show that topographic effects can be significantly affected by the underlying soil stratigraphy; and allude to the potentially significant role of this phenomenon in elevating seismic risk in regions with strong topographic relief. The findings of this study also suggest that future studies will benefit from clear understanding and careful considerations of capabilities and limitations of different investigation methods and that the numerical modeling and the lab testing (or the field testing) methods should complement each other.
\end{abstract}

Keywords: topography, finite elements, centrifuge experiments, boundary effects, amplification

\section{Introduction}

The modification of seismic ground motion by ground surface features such as hills, ridges, cliffs, and canyons, known as topographic effects, is a well documented phenomenon [26]. Observations from past earthquakes have systematically shown that steep slopes or complicated topographies accentuate earthquake damage compared to flat ground. Examples such as the $P G A=1.82 \mathrm{~g}$ ground motion recording on the Tarzana hilltop during the 1994 Northridge Earthquake [7], the Pacoima Dam $(P G A=1.12 g)$ recording during the 1971 San Fernando earthquake [5], and the recent extraordinary ground motion $(P G A=2.74 g)$ recorded at the Japanese Seismic Network K-Net station MYG004 during the 2011 Tohoku Earthquake [8], are only a few of many records that have been linked to topographic effects.

Field studies have confirmed the observational evidence that surface topography significantly affects the amplitude and fre-

\footnotetext{
* Corresponding Author

Email address: seokho.jeong@waikato.ac.nz (Seokho Jeong)
}

quency content of surface ground motion [4, 9, -12]. These studies, however, have been based almost exclusively on ground motions from small or distant events, and weren't accompanied by detailed models of subsurface stratigraphy and dynamic soil properties that one could use to simulate the field observations.

Topographic effects have been extensively studied using techniques such as closed form analytical solutions, finite differences, finite elements and boundary elements [3, 5, 6, 11, 1322]. More recently, large-scale numerical simulations have been used to study realistic geometries of larger features, for which researchers used high resolution digital elevation maps to extract the three-dimensional geometry [22--24].

Despite the qualitative agreement between simulations and observations, however, theoretical predictions largely underestimate observations of topographic amplification compared to field data, by a factor of 10 or more in some cases [11]. This discrepancy has been attributed, at least in part, to idealizations made in published studies, such as the assumption that topographic features lie on homogeneous, elastic soils, and that monochromatic pulses can capture the topographic amplification observed during earthquakes [21]. 
Recently, a series of studies have highlighted the important role that shallow material heterogeneities may be playing on the observed amplification of ground motions on elevated sites [25[29]. Results from these studies, however, may not be representative of topographic effects induced during large earthquakes, which frequently trigger nonlinear response of the shallow geologic layers: recorded topographic amplification factors from well-documented case studies are available primarily for weak and moderate events [29-33], while most published numerical predictions are valid for linear viscoelastic media.

To investigate the extent to which subsurface geology characteristics affect topographic effects, Dafni [1] recently conducted a series of centrifuge experiments. The experimental data can be found in Dafni and Wartman [34]. The experiments involved simple slope features of various geometries subjected to ground motion suites that ranged from weak to strong -adequate to cause nonlinear effects, but not strong enough to cause slope stability failure; and frequency contents from monochromatic to broadband.

In this study, we used results from Dafni [1] to understand whether centrifuge experiments capture the expected characteristics of the topographic effects, and specifically, whether wave reflections caused by the centrifuge laminar box boundaries were strong enough to qualitatively alter the amplification pattern compared to simulated results without the container boundary. Using finite element simulations validated by a subset of centrifuge observations, we studied changes in the ground surface response near the peak that were induced by removing the lateral and vertical constraints (the laminar box and aluminum base plate) in our numerical model, while keeping the geometry of the topographic feature constant. Results and interpretation thereof in the context of field observations of topographic effects

The scope of our study was twofold: (i) to highlight the advantages and limitations of centrifuge experiments in simulating topographic effects, and in doing so, to guide the design and interpretation of future experimental studies on 2D and 3D site effects; (ii) to provide a detailed description of a numerical model of free-field nonlinear topographic effects that we, and other researchers, can use to expand our understanding for this class of problems.

\section{Validation: Numerical simulation of centrifuge experi- ments}

Dafni [1] conducted model test experiments at $27 \mathrm{~g}$ and $55 \mathrm{~g}$ acceleration at the centrifuge facility of the University of California, Davis. The models were prepared with dry Nevada sand in the flexible shear beam container of the facility (FSB-2), which comprises an aluminum base plate and five metal rings. The rings are separated by $12 \mathrm{~mm}$ thick soft neoprene rubber layers that provide lateral flexibility. Technical specifications of the centrifuge, shaking table (base plate) and containers at the U.C. Davis experimental facility can be found in Kutter et al. [35].

\subsection{Geometry, boundary conditions and material calibration}

We simulated the centrifuge experiments using the finite element (FE) code DYNAFLOW ${ }^{\mathrm{TM}}$ [36]. Of the three single slope configurations $\left(20^{\circ}, 25^{\circ}\right.$ and $\left.30^{\circ}\right)$ tested by Dafni [1] we next present numerical predictions for the $30^{\circ}$ single slope. Since topographic amplification is proportional to the feature steepness, and slope instability of granular media increases as the natural slope approaches the material friction angle, the $30^{\circ}$ slope was selected as the 'worst case' scenario configuration among all single slopes tested. We also simulated the flat ground model of Dafni [1] that we used as reference site for flat ground response (Figure 1). The finite element meshes of the two models are shown in Figure 2

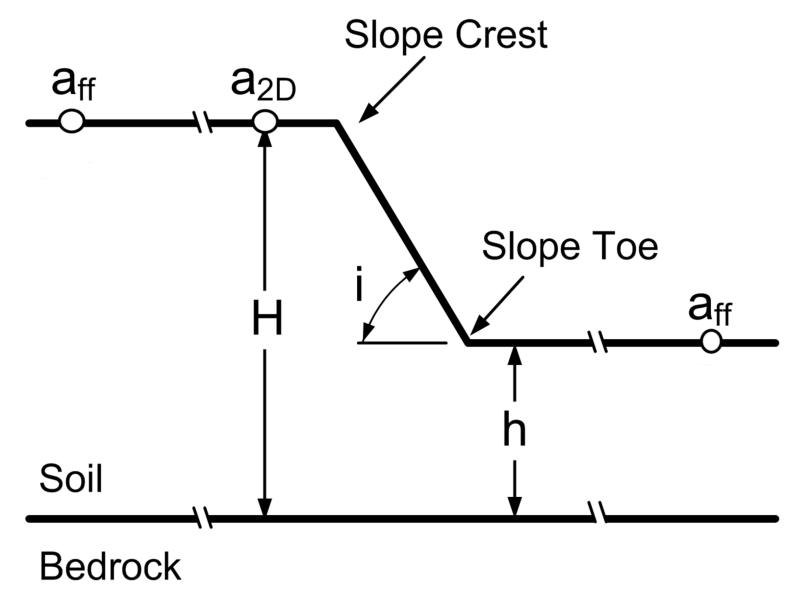

Figure 1: Nomenclature for studies on topographic effects: near-crest acceleration, $a_{2 D}$, is affected by both 1-D site response and topography effect; and free-field acceleration, $a_{f f}$, is affected only by 1-D site response.

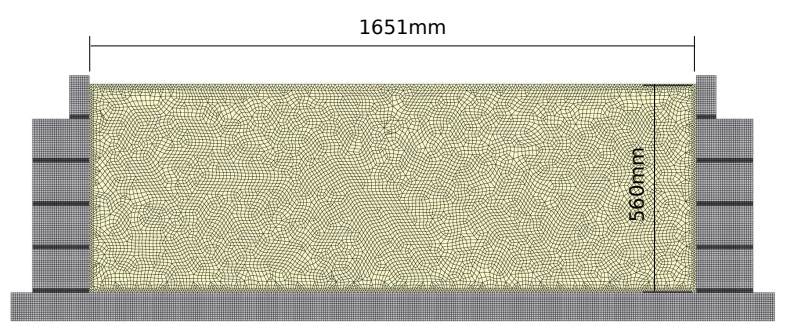

i.

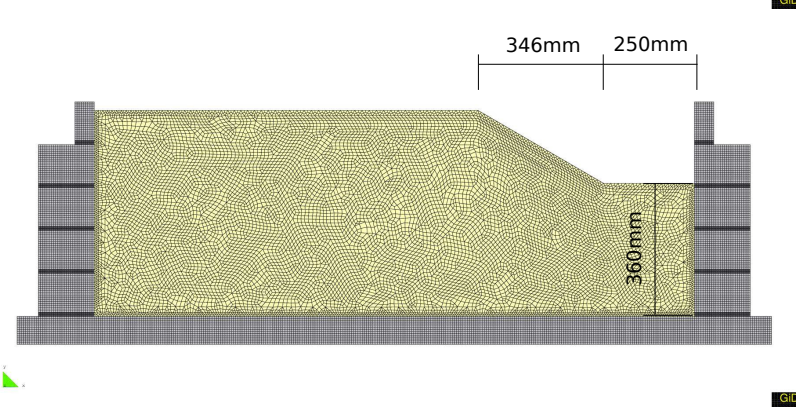

Figure 2: 2-dimensional finite element mesh of a centrifuge experiment model: a flat ground configuration (top) and a single slope with angle of $30^{\circ}$ (bottom); dimensions shown are in actual model scale. 
We used plane strain elements (2D) to simulate the centrifuge box and the soil, and calibrated the mass and stiffness of the 2D numerical model of the container to match the corresponding properties of the three-dimensional (real) container. The linear elastic properties that were selected for the metal rings are described in detail in Kutter et al. [35], and the equivalent material properties of the 2D container are listed in Table 1 .

The neoprene foam rubber that connects the rings was modeled as viscoelastic material. Using the experimental data published by Snowdon [37], we estimated the shear modulus $(G)$ and damping $(\zeta)$ of neoprene rubber at the ambient temperature of the experimental facility as $G=0.25 \mathrm{MPa}$ and $\zeta=6 \%$, respectively. Snowdon [37] also showed that the damping has a mild frequency dependency (i.e. the critical damping ratio reaches ten percent at $f=600 \mathrm{~Hz}$, or $f=11 \mathrm{~Hz}$ in the prototype scale of this study). The Rayleigh damping parameters, $\alpha$ and $\beta$, that we selected to simulate the energy dissipation of the neoprene rubber as measured by Snowdon [37] are listed in Table 1 .

Table 1: Material parameters selected for the equivalent 2D numerical model of the flexible container FSB-2

\begin{tabular}{c|c|c|c|c|c}
\hline \hline & E [MPa $]$ & $v$ & $\rho\left[M g / m^{3}\right]$ & $\alpha$ & $\beta$ \\
\hline Ring 1 & 70000 & 0.35 & 1.92 & 0 & 0 \\
Ring 2 & 70000 & 0.35 & 2.19 & 0 & 0 \\
Ring 3 & 70000 & 0.35 & 2.19 & 0 & 0 \\
Ring 4 & 70000 & 0.35 & 4.14 & 0 & 0 \\
Ring 5 & 70000 & 0.35 & 5.24 & 0 & 0 \\
Rubber 1 & 2 & 0.499 & 2.0 & 9 & 0.0001 \\
Rubber 2 5 & 1.5 & 0.499 & 2.0 & 9 & 0.0001 \\
\hline \hline
\end{tabular}

* The mass densities of the container rings are not of the actual material, but of the equivalent $2 \mathrm{D}$ model.

We rigidly linked the horizontal degrees of freedom of the ring elements, so that the left and right container sides move horizontally in sync; the implicit assumption here is that the axial deformation of the rings during shaking is negligible.

We simulated the dry Nevada sand using the pressure dependent multi-yield (PDMY) plasticity model by Prevost [36. [38]. The model is implemented in DYNAFLOW ${ }^{\mathrm{TM}}$, and employs a purely kinematic hardening rule with nested round-cornered Mohr-Coulomb yield surfaces, an associated flow rule for the deviatoric strains, and a non-associated flow rule for the volumetric plastic strains. The PDMY model has two options for modeling the monotonic shear stress-strain backbone curve: (a) the modified hyperbolic model by Kondner [39], and (b) Hayashi's model [40], a generalization of the Kondner's model. Lastly, the model employs Masing's hysteresis rule [41] for unloadingreloading cycles.

To simulate the response of dry sand using the PDMY model, the only relevant parameters are the initial (elastic) modulus $(G)$, the friction angle $(\phi)$ that governs the ultimate strength, and the backbone curve (or integral of the shear modulus degradation curve). The pressure-dependency of the shear modulus was idealized by the power law of Equation (1), where $G_{0}$ is the elastic shear modulus at the reference hydrostatic pressure $p_{\text {ref }}$.

$$
G=G_{0}\left(\frac{p}{p_{\text {ref }}}\right)^{n}
$$

Figure 3 shows the shear wave velocity $\left(V_{S}\right)$ profile of dry Nevada sand with relative density $D_{r}=100 \%$ measured by Stevens et al. [42] in a previous centrifuge experimental study that used the same container. We used the Stevens et al. [42] measurements for the initial calibration of Equation (1), which we subsequently re-calibrated using ground motion data recorded by Dafni [1] during the flat ground model shaking tests. It turned out that the final velocity profile we used for the simulation differs by $14 \%$, compared with the original profile by Stevens et al. [42]. The difference could be due to the different batch of the sand, or different person preparing the model. Another possibility is that the measurement by Stevens et al. (1999) is done by measuring the air hammer signals before the shaking begins, whereas our calibration is done after shaking the model at low amplitude, which could cause a very dense sand to dilate slightly.

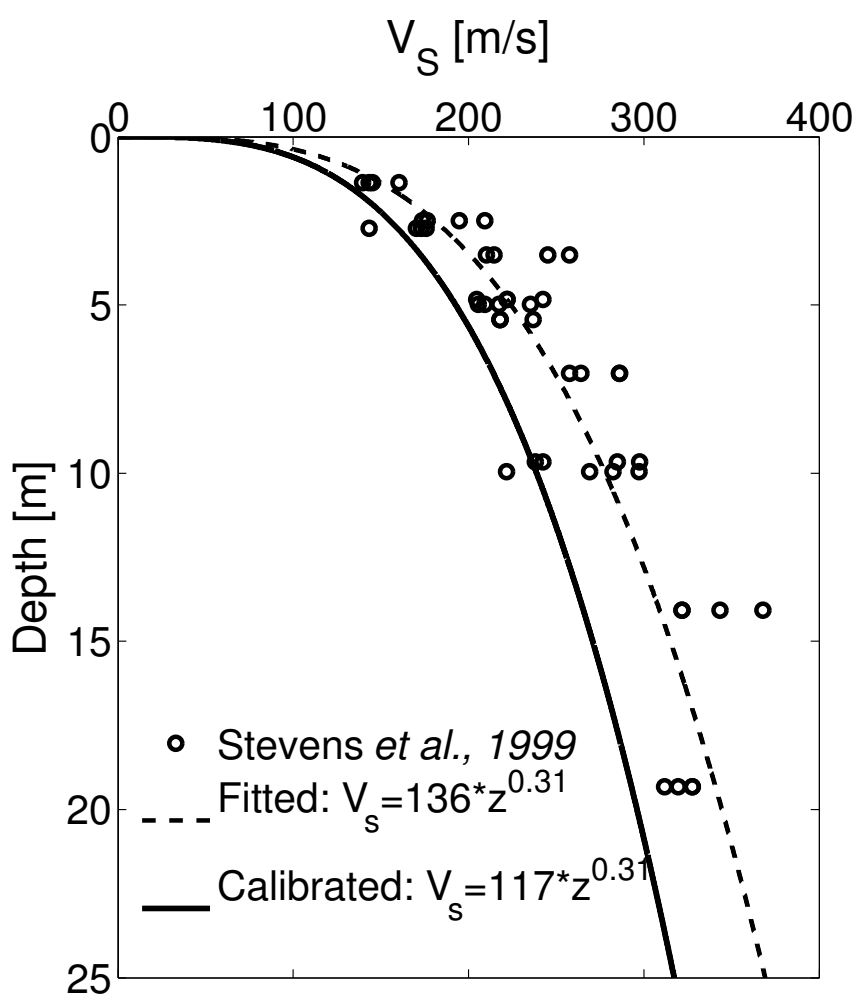

Figure 3: Shear wave velocity profile of dry Nevada sand with relative density of $100 \%$ as published by Stevens et al. [42]. The black dashed line shows fitted power law used in the design phase of the centrifuge experiments; the black solid line shows the final velocity profile after calibration using the result from flat ground model.

Finally, we simulated the monotonic shear stress-strain response of the soil using the generalized hyperbolic model by Hayashi et al. [40] shown in Equations (2) and (3):

$$
\begin{gathered}
f(x, n)=\frac{\left(\frac{2}{n} x+1\right)^{n}-1}{\left(\frac{2}{n} x+1\right)^{n}+1} ; n>0 \\
y=e^{-\alpha x} \cdot f\left(x, n_{L}\right)+\left(1-e^{-\alpha x}\right) \cdot f\left(x, n_{U}\right)
\end{gathered}
$$


where $x$ in our case is the shear strain $(\gamma)$ normalized with respect to the reference strain $\gamma_{r}=\tau_{f} / G_{\text {max }}, \tau_{f}$ and $G_{\text {max }}$ are the soil shear strength and maximum shear modulus respectively, $y$ is the shear stress $\tau$ normalized with respect to the shear strength, $y=\tau / \tau_{f}$, and $\alpha, n_{L}$ and $n_{U}$ are three model parameters. Hayashi's model combines two standard hyperbolic models [39] as shown in Equation (3), and reduces to the standard hyperbolic form when $n_{L}=n_{U}=1$. Such a versatile formulation was necessary to allow simultaneous matching of the soil's shear modulus $\left(G_{\max }\right)$ (at low strains), and ultimate shear strength $\left(\tau_{f}\right)$ (at high strains).

For the model calibration, we used $G_{0}$ and $n$ from the $V_{s}$ data shown in Figure 3 , and assumed a friction angle of $\phi=42^{\circ}$ for the dry Nevada sand with $D_{r}=100 \%$ to estimate $\tau_{f}$. We then calibrated the parameters $\left(\alpha, n_{L}, n_{U}\right)$ for Equations (2) and (3), such that the modulus reduction curve $\left(G / G_{\max }\right)$ of the model is in a good agreement with the existing model by Darendeli [43], while ensuring that the modulus reduction curve is consistent with the estimated $\tau_{f}$. Figure 4 shows the modulus reduction and the damping curves of the calibrated model, in comparison with the model by Darendeli [43]. The PDMY model itself does not dissipate any energy unless the material stress reaches the first yield surface. Therefore, the hysteretic damping is zero when the strain is very small (i.e. $\gamma<10^{-3} \%$ in Figure 4. However, such lack of small-strain damping causes spurious low-amplitude oscillation. In practice, this issue is often avoided by incorporating some other type of damping, and the Rayleigh damping is perhaps the most popular choice in the engineering community due to the simplicity of its formulation. The global Rayleigh damping matrix was not implemented in DYNAFLOW $^{\mathrm{TM}}$, and therefore we instead utilized the algorithmic damping inherent to the Newmark- $\beta$ method to suppress the spurious oscillation [44, 45], such that the global damping of the model is approximately equivalent to the stiffnessproportional (i.e. linearly increasing with frequency) damping with $10 \%$ of damping ratio at $f=10 \mathrm{~Hz}$. The median damping in $f \leq 6 \mathrm{~Hz}$ is therefore $3 \%$, and later it will be shown that the simulated motions have negligible energy in $f>6 \mathrm{~Hz}$. Figure 4 also shows that our model has significantly higher damping ratio than Darendeli's model when the strain is larger than $0.1 \%$. Such overprediction of the damping is a well-documented drawback of the Masing's rule [43], and therefore it should be realized that the simulated response could be affected when the shear strain is large enough. The soil parameters that were used in the simulations described in the following sections are summarized in Table 2.

Lastly, we should mention here that we assumed perfect contact between the laminar box and the soil elements. There are a few different approaches with various levels of complexity for modelling the interface between the soil and the container. For example, several published studies [46, 47] ignored the container in their numerical models, assuming the periodic boundary condition, which is the ideal case the laminar box is designed to simulate. Or one could go to the opposite extreme by modeling the soil and container in full 3D, including the container-soil contact model. Of course, our approach, which we consider a good compromise between the reality and

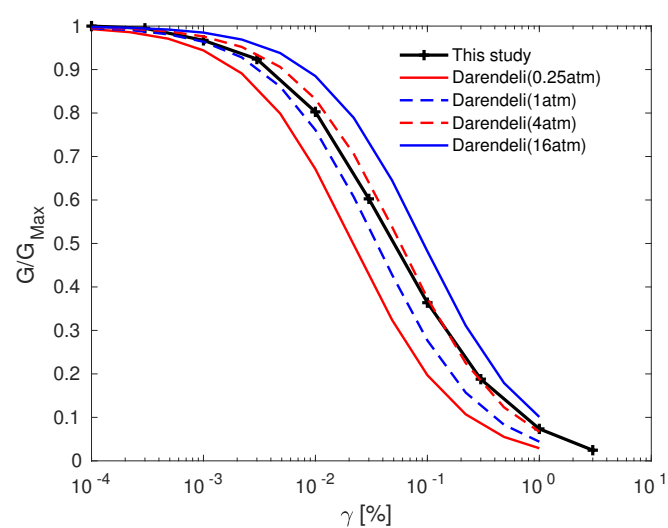

(a) Modulus reduction curve

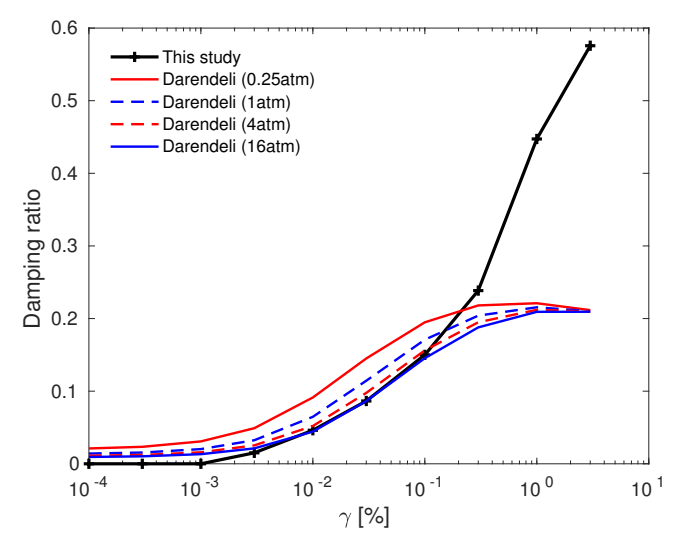

(b) Damping curve

Figure 4: (a) Calibrated modulus reduction curve and (b) the damping curve at $p_{\text {ref }}=100 \mathrm{kPa}$ compared to the data by Darendeli [43.

the simplicity, is still an approximation of a complex behavior, and therefore cannot simulate localized highly nonlinear effects, such as the local shear failure of the sand induced by the laminar box shaking due to the instant loss of confinement and subsequent container-soil pounding at the top ring's displacement reversals. However, we were able to approximate this effect by using the PDMY constitutive soil model with zero tensile strength, which captured failure caused by lack of confining stress in the shallow layers of the soil-container interface, as will be shown in the following sections.

\subsection{Simulated ground motions}

The ground motions we selected from Dafni [1] for our numerical simulations are listed in Table 3, they include three recorded (broadband) ground motions (BPTS315, JOS090 and TCU078E), each scaled at two different amplitudes; a frequency sweep (FREQ55NEW); and a sequence of Ricker wavelets (RICK55). The time histories and corresponding Fourier amplitude spectra, as recorded at the baseplate of the container, are plotted in Figure 5 .

\subsection{Validation of simulated ground motions}

Results from the simulated experiments were next compared to recorded motions, which are here presented in prototype units 
Table 2: Material parameters of the PDMY model calibrated for dry Nevada sand at relative density $D_{R}=100 \%$ and used in the simulations presented in this paper

\begin{tabular}{c|c|c|c|c|c|c|c|c|c|c}
\hline \hline$\rho\left[\mathrm{Mg} / \mathrm{m}^{3}\right]$ & $v$ & $G_{0}[\mathrm{MPa}]$ & $p_{\text {ref }}[\mathrm{kPa}]$ & $\mathrm{n}$ & $\phi\left[^{\circ}\right]$ & $\Psi\left[^{\circ}\right]$ & $X_{p p}$ & $\alpha$ & $n_{L}$ & $n_{U}$ \\
\hline 1.7 & 0.25 & 108 & 100 & 0.62 & 42 & 35 & 0.05 & 0.4 & 0.5 & 1.0 \\
\hline \hline
\end{tabular}
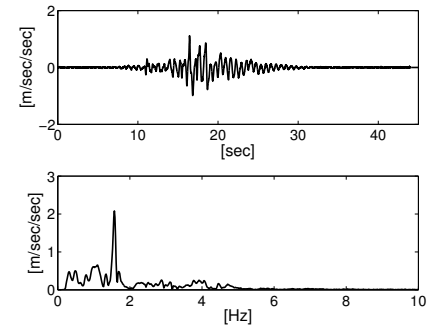

(a)BPTS315 @ 0.5
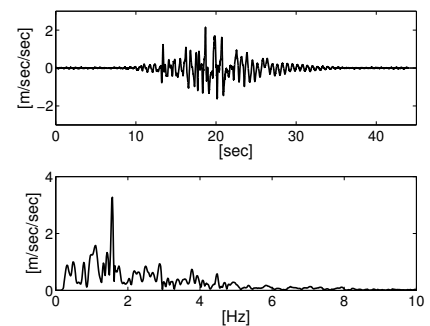

(e) BPTS315 @ 1
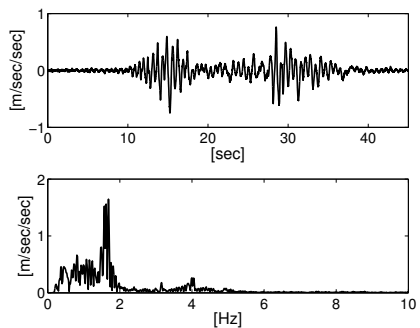

(b) JOS090@0.3
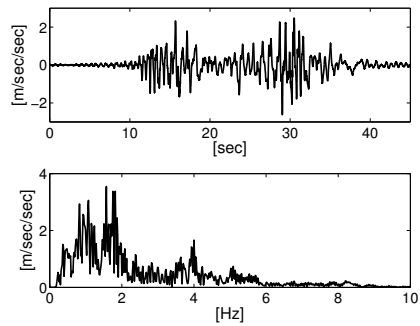

(f) JOS090@1
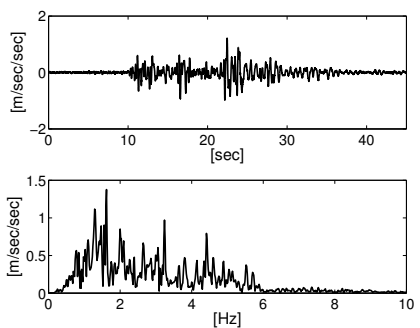

(c) TCU078E@0.7
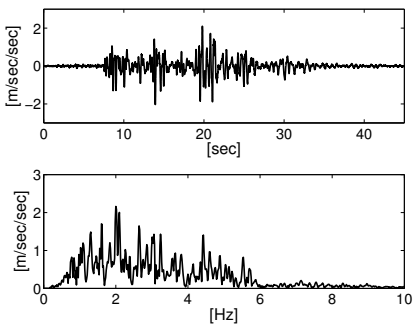

(g) TCU078E @ 1
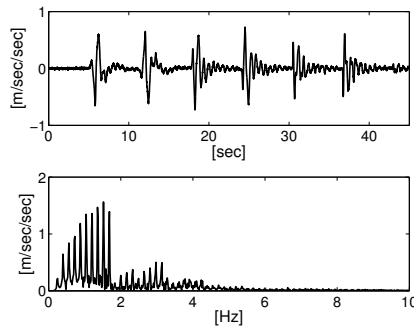

(d) RICK55 @ 1
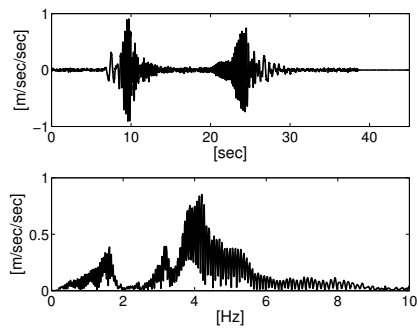

(h) FREQ55NEW @ 1

Figure 5: Baseplate acceleration time histories and Fourier amplitude spectra, used as input motions for numerical simulations.

unless otherwise stated. We first compared recordings from the surface sensors A28-A29 located in the off-plane direction along the centerline (see Figure 6; it was important to examine whether the ground surface response was contaminated by boundary effects normal to the direction of shaking, because such a finding could challenge our assumption of plane strain conditions.

Ground motion variability was absent from the recordings in the off-plane direction, as shown in Figure 7, which compares simulated and recorded acceleration time histories of the flat model subjected to the BPTS315, JOS090, and RICK55 motions (for code names refer to Table 3). The corresponding Fourier amplitude spectra are shown in Figure 8 . We then compared simulated to recorded motions along the vertical array of sensors at the centerline of the container (see sensors A29, A56, A55, A54 and A53 in Figure 8). Results for the $30^{\circ}$ slope are shown in Figure 9. Overall, simulated and recorded ground motions showed good agreement in a wide range of frequencies for the most ground motions considered.

\subsection{Nonlinear response of the soil model}

The physical model we tested in the geotechnical centrifuge is expected to exhibit a significant nonlinear response, when it is subjected to strong shaking. Understanding the level of such nonlinear response is helpful for interpreting the experimental data and the result of numerical simulations. However, measuring directly the dynamic shear strain and stress of the soil in the centrifuge container is not feasible in general. We therefore obtained the shear stress-strain curves from the numerical model, along the centerline of the model at the locations of sensors A29, A56, A55, A54 and A53, as shown in Figure 10 for three selected ground motions: BPTS315 scaled by 0.5, RICK55unscaled and TCU078E-unscaled. The simulated stress-strain curves suggest that the maximum shear strain is in the order of $0.1 \%$, which can be considered moderate or moderately large. Figure 4 suggests $G / G_{\text {Max }}$ ratio of 0.38 and the damping ratio of 0.15 at this strain level. Figure 10 also shows that the shear strain of 0.1 is reached for a few largest cycles (except for RICK55, for which the strain amplitude never reached $0.1 \%$ ), and there are many cycles of shear strain with amplitude less than $0.03 \%$, which corresponds to $G / G_{\max }$ ratio of 0.6 and the damping ratio of 0.09 .

\subsection{Goodness-of-fit evaluation}

To quantify the goodness-of-fit (GOF) of simulated motions to the recorded data, we chose the wavelet based method by Kristeková et al. [48], which defines the GOF criteria in terms of both time-frequency amplitude envelope and phase misfit of observations and simulations. Figure 11 shows an example of the said time-frequency GOF evaluation for the slope model, subjected to the motion BPTS315 scaled by 0.5 , using the Python Obspy package [49].

Results from this simulation yielded a GOF envelope with peak value $E G=7.47$, and phase GOF value $P G=7.68$. The higher the GOF, the better the match between observations and simulations, with 10 indicating perfect fit between the two. According to Kristeková et al. [48], the EG and PG of this simulation lie in the GOF range between "GOOD" and "EX- 

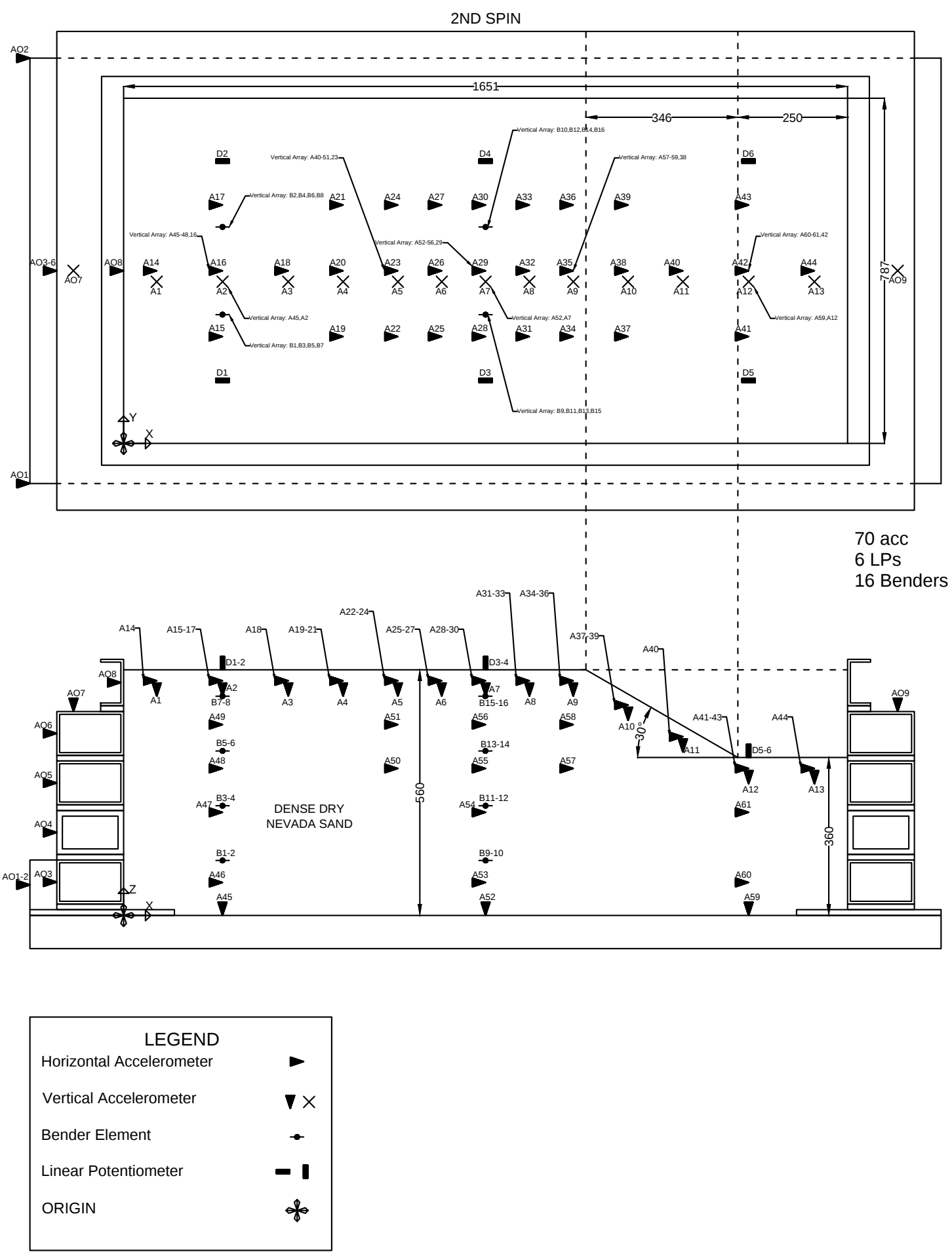

Figure 6: Sensor deployment plan for the centrifuge model with $30^{\circ}$ slope. Dimensions are in millimeters. 
Table 3: Ground motions selected for numerical simulations

\begin{tabular}{c|c|c|c|c|c}
\hline \hline Label & Description & $\begin{array}{c}\text { Prototype } \\
\text { amplitude }(\mathrm{g})\end{array}$ & $\begin{array}{c}\text { Amplitude } \\
\text { scale }\end{array}$ & $\begin{array}{c}\text { Moment } \\
\text { magnitude }\end{array}$ & $\begin{array}{c}\text { Distance, } \\
R_{\text {Rup }}(\mathrm{km})\end{array}$ \\
\hline BPTS315 & Superstition Hills-Parachute T S 315 & 0.25 & 0.5 and 1 & 6.5 & 1.0 \\
JOS090 & Landers-Joshua Tree 090 & 0.25 & 0.3 and 1 & 7.3 & 11.0 \\
TCU078E & Chi Chi-TCU078E & 0.1 & 0.7 and 1 & 7.6 & 8.2 \\
FREQ55NEW & Frequency Sweep 1-7-1Hz & 0.1 & 1 & - & - \\
RICK55 & Ricker wavelets 1-2-4-6Hz & 0.1 & 1 & - & - \\
\hline
\end{tabular}

BPTS315: Scale $=0.5$

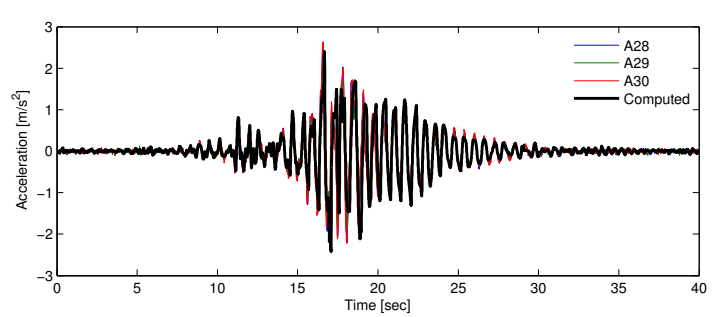

JOS090: Scale $=1$

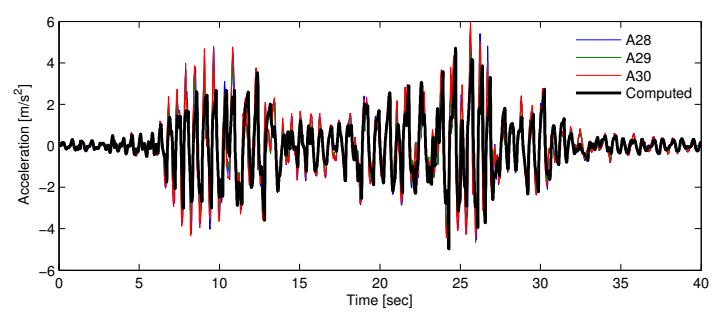

RICK55: Scale $=1$

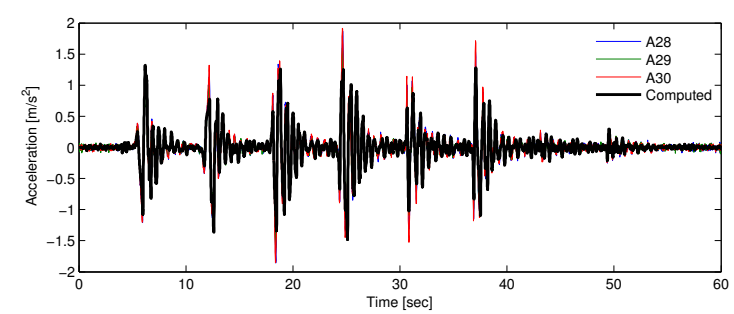

Figure 7: Validation of numerical model: Computed acceleration time histories from the $30^{\circ}$ single slope model at the center of the surface compared with the centrifuge experiment results measured from sensors A28, A29 and A30.
BPTS315: Scale $=0.5$

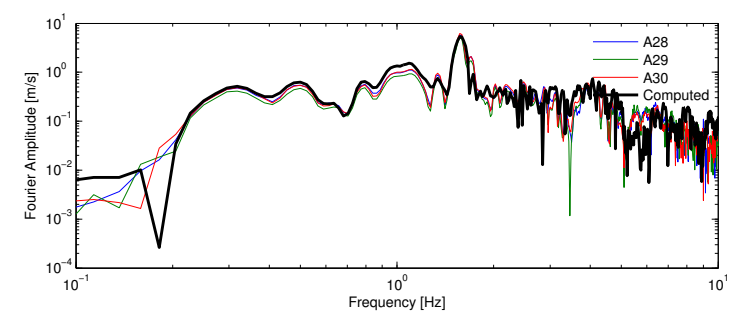

JOS090: Scale $=1$

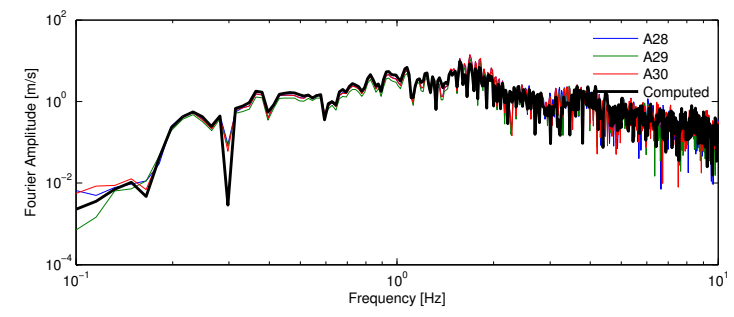

RICK55: Scale $=1$

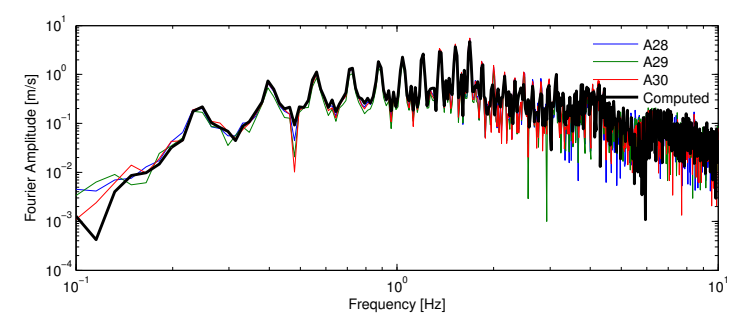

Figure 8: Validation of numerical model: Fourier spectra of computed acceleration time histories from the $30^{\circ}$ single slope model at the center of the surface compared with the centrifuge experiment results measured from sensors A28, A29 and A30. 
BPTS315: Scale $=0.5$

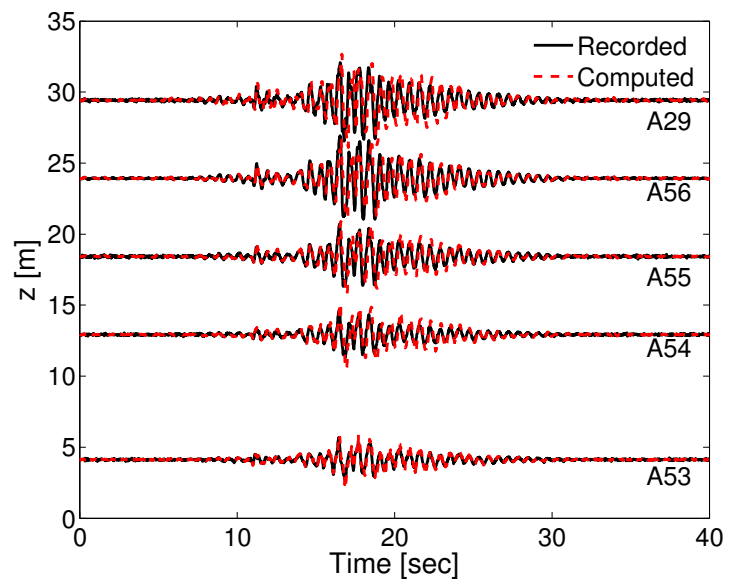

Rick55: Scale $=1$

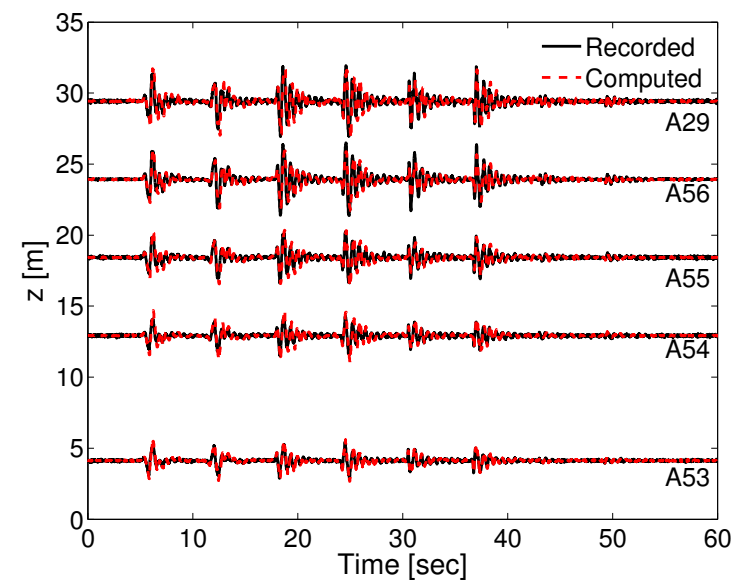

Figure 9: Validation of numerical model: Arrays of horizontal acceleration time histories computed along the depth of the numerical model (solid line) from the $30^{\circ}$ single slope model compared to the corresponding experiment results measured at sensors A29, A56, A55, A54 and A53 (dashed line).
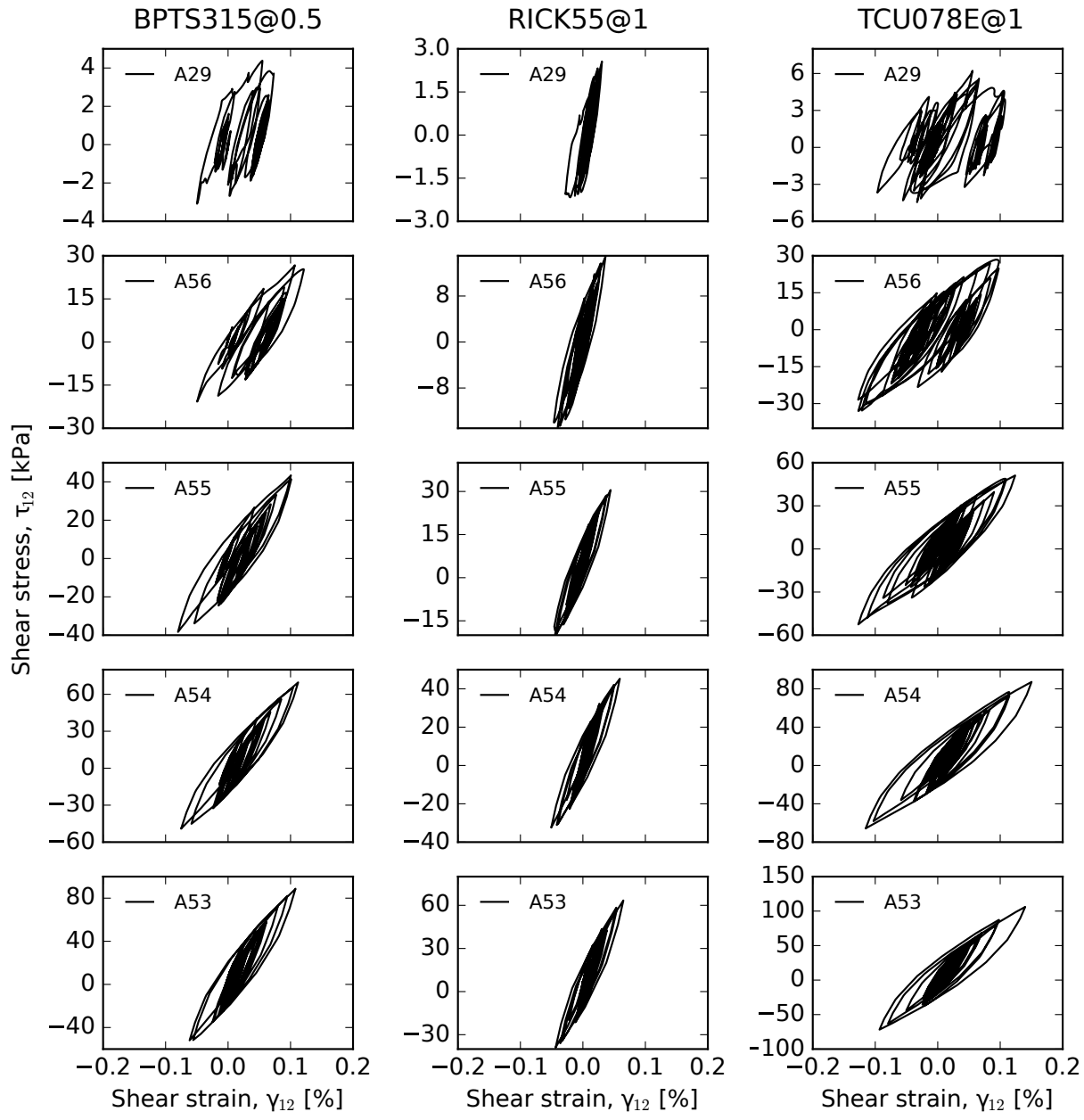

Figure 10: Simulated stress-strain curves along the centerline of the model, at the locations of sensors A29, A56, A55, A54, and A53, for motions BPTS315 scaled by a factor of 0.5 , RICK55-unscaled, and TCU078E-unscaled. 
CELLENT". Most simulations that we conducted were found to classify in the same category.

Figure 12 shows the envelope and phase misfit values as function of frequency, for all motions considered in this study. Despite some inevitable uncertainty, the misfit from different simulations are broadly consistent, and mostly fall within the GOF "EXCELLENT" category.

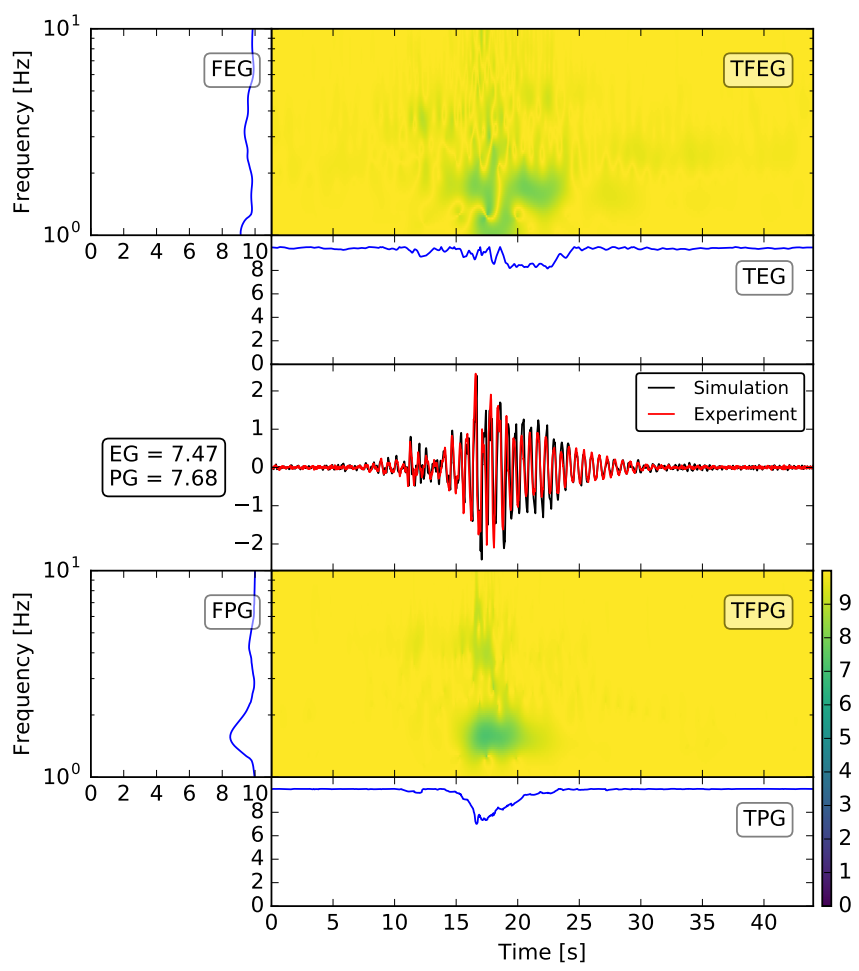

Figure 11: Time-frequency goodness-of-fit (GOF) representation of the slope model simulation, subjected to the motion BPTS315 scaled by 0.5 , evaluated using the approach by Kristeková et al. [48], implemented in Obspy [49]. In the abbreviations for different criteria, EG and PG stands for the "envelope GOF" and "phase GOF", and T and F stands for "time" and "frequency", respectively.

\subsection{Topographic amplification factors: Empirical vs. Com- puted}

Traditionally, topographic effects have been quantified as the modification (typically amplification) of ground motion in excess of 1D site response [17, 21]. Previously published studies on topographic effects have specifically used the ratio of the Fourier amplitude spectrum of ground motion at (or near) the crest to the Fourier spectrum of the motion in the free-field, as shown in Equation (4):

$$
S R(\omega)=\frac{A_{2 D}^{h}(\omega)}{A_{f f}^{h}(\omega)}
$$

where $A(\omega)$ is the Fourier amplitude of acceleration time history $a(t), \omega=2 \pi f$ is the circular frequency, and the superscript $h$ refers to the horizontal component of ground motion.

For compatibility with previously published results, we here evaluated topographic amplification using that same ratio (SR).
However, we used the flat ground model response as representative of the free-field ground motion instead of the flat sections of the sloped model (see stations A16, A18 or A20 for example in Figure 6, to avoid recordings possibly contaminated by reflections of surface waves caused by diffraction at the toe and crest of the sloped models.

As mentioned above, the premise of Equation (4) is that the stations that record $a_{2 D}(t)$ and $a_{f f}(t)$ have the same soil profile and the same input motion. Satisfying the latter condition in centrifuge experiments, however, is not a trivial task. Specifically in this case, while the input (command) displacements of the flat ground and sloped tests were the same, the total (recorded) baseplate motions were different because of the differences in mass (and thus inertia feedback) of the two configurations. To correct the experimental topographic amplification factor for the baseplate motion difference, we used the theory of reciprocity as shown in Equation (5):

$$
\hat{S R}(\omega)=\frac{A_{2 D}^{h}(\omega)}{A_{f f}^{h}(\omega)} \times \frac{A_{B P, \text { flat }}^{h}(\omega)}{A_{B P, \text { sloped }}^{h}(\omega)}=\frac{A_{2 D}^{h}(\omega)}{\hat{A}_{f f}^{h}(\omega)}
$$

where $\hat{A}_{f f}^{h}(\omega)$ is the corrected free-field reference ground motion, computed by convolving the recorded baseplate motion of the sloped model with the experimental 1D transfer function (surface over baseplate) of the flat ground model; BP stands for baseplate, flat for the flat ground model, and sloped for the sloped model. The median and $68 \%$ confidence interval of the correction term in equation (5) is shown in Figure 13, which indicates that -although the command motion of the two series of experiments was the same, as mentioned above- the difference in spectral amplitude of the recorded baseplate motions was as high as $50 \%$ for frequencies in the range $f=1.5-6 \mathrm{~Hz}$. We should mention here that Equation (5) is technically valid only for weak ground motions or linear elastic analyses, but its applicability can be extended to weakly nonlinear systems. In the simulated experiments on the other hand, we prescribed the exact same base plate motion for sloped and flat ground, so this correction was not necessary. Figure 14 compares the corrected experimental to the computed median topographic amplification factor at the crest (station A35).

Results showed that topographic amplification manifested at approximately the same frequencies in both cases, $3.5 \mathrm{~Hz}$ and $6.5 \mathrm{~Hz}$. The first frequency peak, at $f=3.5 \mathrm{~Hz}$, is consistent with what Ashford et al. [17] defined as topographic frequency. Their study showed that for idealized single slope configurations such as the one tested here, the maximum topographic amplification occurs at frequency $f=V_{S} / 5 h$, where $h$ is the slope height. Currently, we do not have a clear explanation about the second peak at $6.5 \mathrm{~Hz}$. However, considering that many previous studies have not reported such second peak, relatively close to the first peak, it is possibly related to the complex wave interaction specific to the model considered in this study.

Although the simulations and experiments agreed well in terms of the frequencies of amplification, the agreement was less favorable for the corresponding absolute peak amplitudes, as shown in the same figure. The mismatch is likely due to inherent limitations of our numerical simulations and centrifuge 


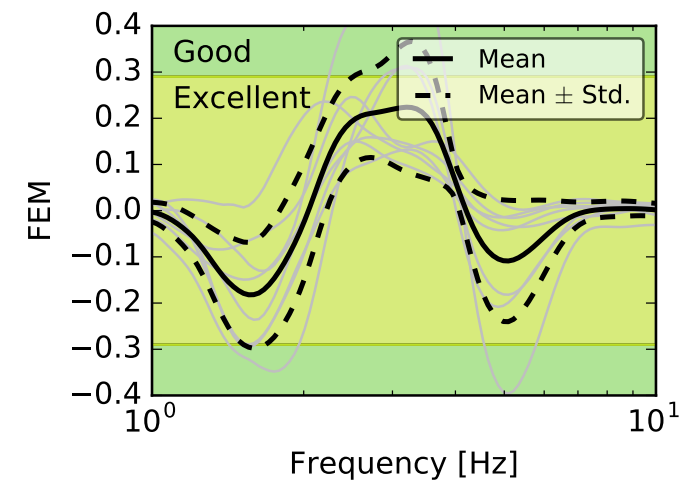

(a) Envelope misfit for the flat model

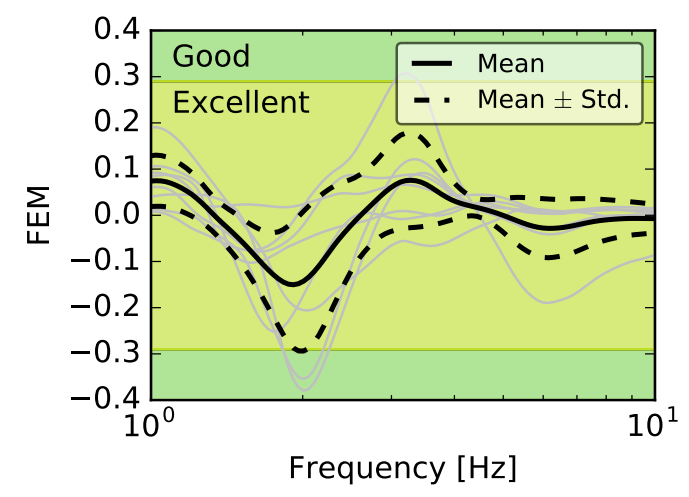

(c) Envelope misfit for the slope model

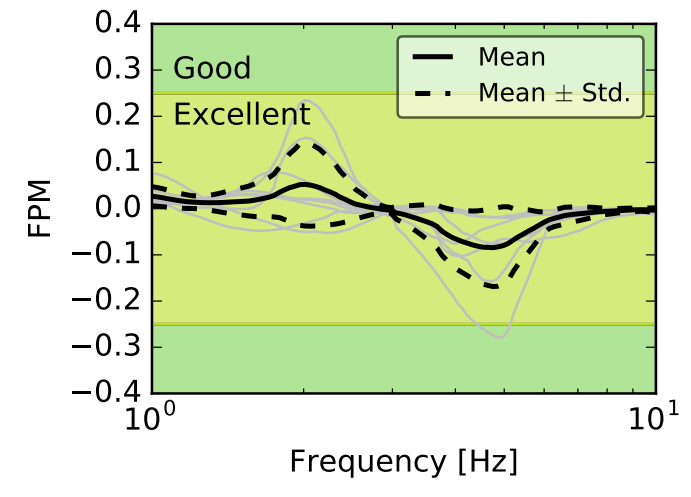

(b) Phase misfit for the flat model

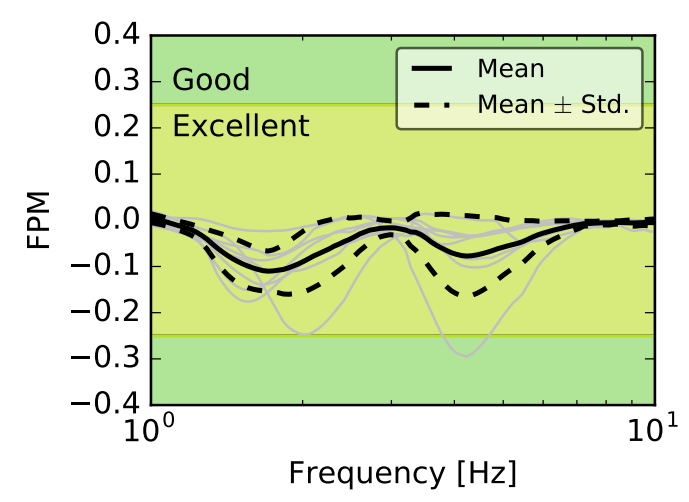

(d) Phase misfit for the slope model

Figure 12: Frequency domain envelope (FEM) and phase misfit (FPM) values for the flat and the slope model. Verbal evaluations are based on the recommendation by Kristeková et al. 48

experimental errors. Examples in the former category include, but are not limited to, the assumption of linear or weakly nonlinear response in the spectral ratio correction that we employed on the experimental data; the contribution of three-dimensional effects and that was not accounted for in our numerical simulations; and the uncertainty in calibration of the constitutive soil model to capture the hysteretic damping. We also found that although the centrifuge test was intended to simulate shear wave incidence, the rocking of the soil-container system was substantial, which could have adversely affected the experimental crest-to-free field spectral ratio. We will be discussing this subject in detail later in the paper.

\section{Boundary effects of the centrifuge container}

Considering that numerical simulations of all sloped models (of which the $30^{\circ}$ is shown here) were found to be in good agreement with the experimental data, we proceeded with the assumption that the computed spectral ratio (solid line in Figure 14) was a realistic prediction of the empirical ratio that would have been measured, had the base plate motions of flat and sloped ground experiments been identical. With this premise, we examined the extent to which: (i) the rocking of the soilcontainer system; (ii) the laminar container lateral boundaries; and (iii) the aluminum base plate (analogous to very hard bedrock in the field condition) affected the experimental results of topographic amplification.

\section{1. 'Rigid-body' rocking of the soil-container system}

The dynamic shaker actuator of the centrifuge experimental facility is designed to prescribe pure sliding motion; rocking of the container-shaking table with respect to the reaction mass, a byproduct of the sliding motion, centrifugal motion of the box and non-symmetry of the soil specimen, is considered undesirable. Thus, while the experimental design is tailored to minimize the rocking motion (e.g. Kutter et al. [35]), some level of the coupled rocking motion is inevitable because of the practical difficulties associated with ensuring the pure sliding motion at the actuator level (i.e. achieving free sliding and zero relative vertical displacement above the base plate would be difficult).

This series of experiments was no exception; we quantified the rocking motion of the soil-container system using the recorded vertical accelerations (A45 and A59 in Figure 6) on the base plate. Assuming that the dynamic (i.e. de-meaned) 


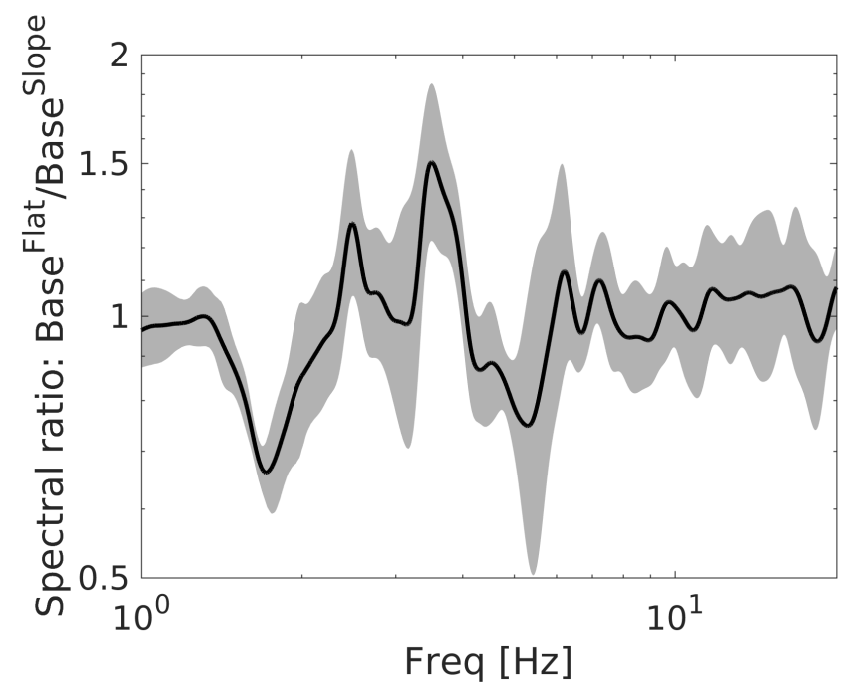

Figure 13: Baseplate-to-Baseplate spectral ratios.

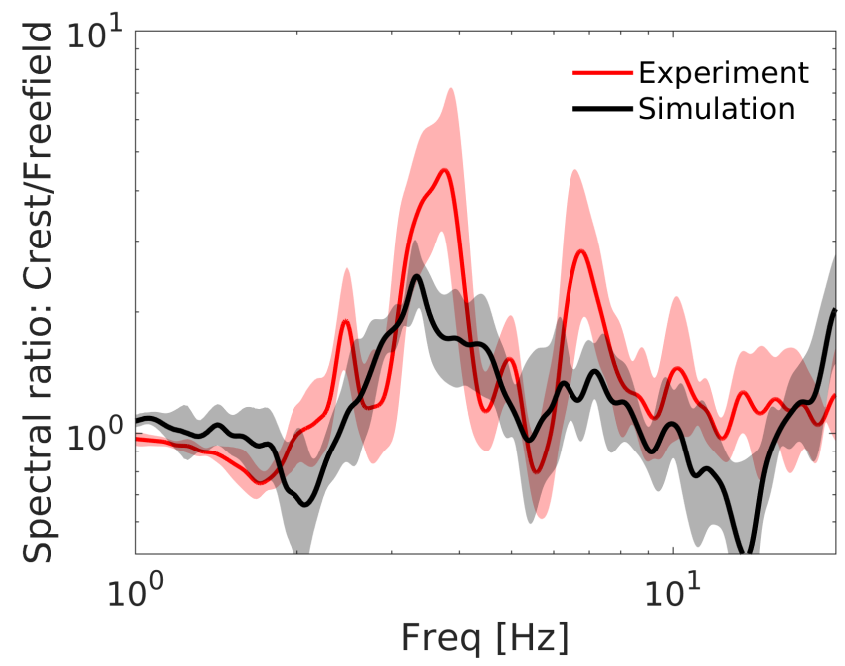

Figure 14: Validation of numerical model: Comparison of crest to freefield median spectral ratio of all considered ground motions

vertical accelerations at those two accelerometers are purely caused by the baseplate rocking, the recorded accelerations of the two vertical accelerometers are expected to be equal and opposite, given that they are installed at equal distance from the center of the model, and the center of rotation is expected to be near the center line of the model. Unfortunately the sensor A59 malfunctioned after a small number of initial spins. Still, under the assumption of rigid-body rocking, the vertical accelerometers A07 and A09 recorded accelerations that were indeed equal and opposite, which strongly suggests that A59 and A45 would have recorded equal and opposite accelerations. We therefore estimated the container rocking acceleration, $\ddot{\theta}$, using A45 only, and assuming that the center of rotation lied approximately along the center line of the model:

$$
\ddot{\theta}(t) \approx \ddot{u}_{z}(t) / \Delta x
$$

in which $\Delta x$ is the distance of sensor A45 from the center (and center of rotation) of the baseplate.

Further assuming that the center of rotation was stationary during the experiment, we estimated the contribution of the rigid-body rocking to the surface horizontal (tangential) acceleration as follows:

$$
\ddot{u}_{x}^{\text {Rocking }}(t)=\ddot{\theta}(t) \Delta z
$$

in which $\Delta z$ is the distance of the surface from the center of rotation.

Figure 15 shows the Fourier amplitude spectra of $\ddot{u}_{x}^{\text {Rocking }}$, estimated on the base of the foregoing assumptions, and normalized by the baseplate motion. Interestingly, we found that the baseplate rocking had a pronounced peak at frequency, $f=$ $3 \mathrm{~Hz}$, which is very close to the topographic frequency revealed by the experiments, $f=3.5 \mathrm{~Hz}$. Figure 15 in fact shows that the amplitude of the baseplate rocking spectrum at $f=3 \mathrm{~Hz}$ -and thus the contribution of rocking to the horizontal motion recorded on the surface- was comparable to the amplitude of the baseplate horizontal motion itself, assuming here that the center of rotation was located on top of the baseplate.

The question that naturally arises next is, "To what extent did the container rocking affect the experimental topographic amplification ratio?" To answer this question, we removed the contribution of rigid-body rocking (as estimated above) from the observed horizontal acceleration at the crest, by subtracting the values of equation (7) from the recorded ground surface motion in the time domain. Figure 16 demonstrates the estimated effect of the container rocking to the crest-to-freefield spectral ratios: it compares the original spectral ratios before and after the rocking has been removed, and clearly shows that the container rocking increased the amplitude of the experimental spectral ratio at the crest by approximately $20 \%$ in frequencies $f=3-4 H z$.

We should stress here, however, that the procedure described in this section to compute $\ddot{u}_{x}^{\text {Rocking }}$ is an approximation based on a series of educated assumptions, and thus Figure 16 should be used only as qualitative-and not quantitative evidence of the effects of rocking. After all, removing the rocking effects did not eliminate the discrepancy between the simulated and measured spectral ratio amplitude, which we still attribute to a number of simplifications of our numerical model described in Section 2 above.

\subsection{Effects of the lateral boundaries}

After studying the effects of container rocking on the measured topographic amplification, we examined the effects of the laminar box lateral boundaries. Flexible containers, like the one used in this study, minimize boundary reflections through a stack of rigid rings [50, 51]. Previous studies showed that for a $1 \mathrm{D}$ profile subjected to $1 \mathrm{D}$ motion, the stacked-ring container models similar to what was used in this study approximate a profile that extends laterally to infinity [50]: since the left and right sides of the model are constrained to move in sync, two models could be stitched in series without changing the problem, then three and so on. Consider now a 1D profile with a scatterer (e.g. a hill, a boulder, a tunnel) subjected to uniform 


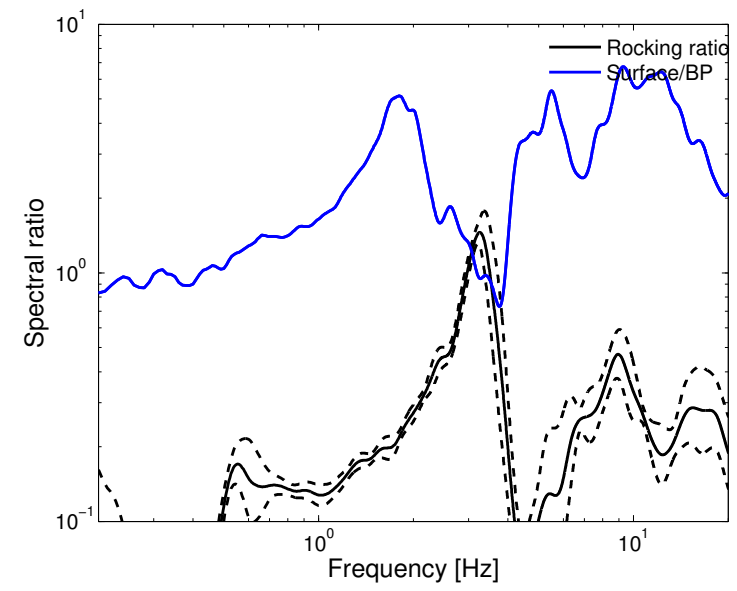

(a) Flat model

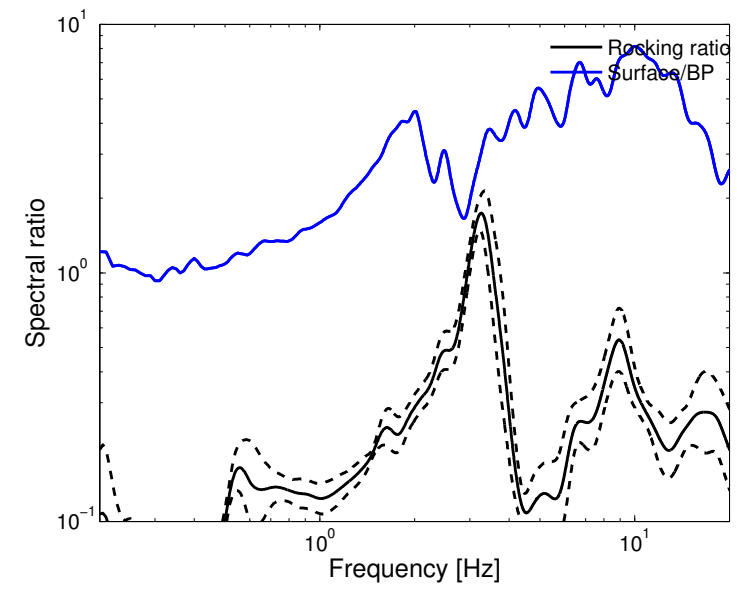

(b) Slope model

Figure 15: Contribution of container rocking to the horizontal acceleration at the surface. The median rocking spectral ratio is shown with the standard deviation of all considered ground motions. The surface-to-baseplate spectral ratio is the median of all considered motions.

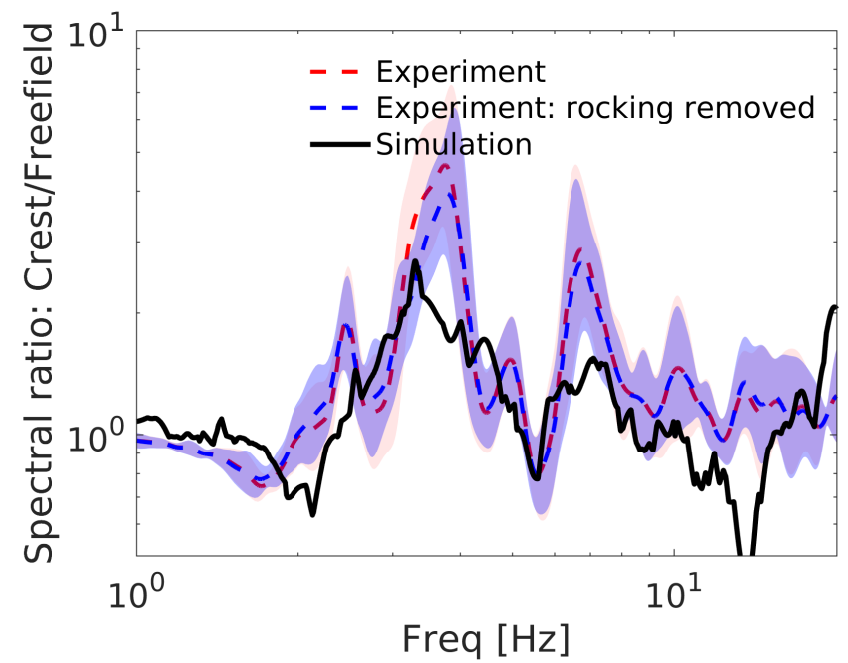

Figure 16: Effect of the container rocking to the crest-to-freefield spectral ratio at the crest. Spectral ratios are the median of all considered motions.

base motion. While the input motion and soil profile are 1D, the wavefield in the vicinity of the scatterer comprises shear, compressional and surface waves, generated by scattering and diffraction and traveling in multiple directions. In turn, if the container boundaries are placed too close to the scatterer, this complex wavefield and the $1 \mathrm{D}$ boundary response would differ and this difference would cause spurious reflections. To avoid this artifact, the container sides should thus be placed $\mathrm{ad}$ equately far from the scatterer, to allow the complex wavefield to dissipate, and the model response to converge to 1D, namely to a motion compatible with the laminar box boundary conditions. Too close and adequately far in this case are not defined in absolute terms: since attenuation is proportional to the number of wavelengths travelled, higher frequency waves attenuate faster than lower frequencies in the same medium, and thus the former require shorter absolute distances to attenuate than the latter.

The configuration tested in the centrifuge comprised a horizontally stratified soil (1D) with the surface geometry acting as a scatterer, and was subjected to approximately (due to container and baseplate rocking) uniform horizontal base motion (also see Dafni [1]). Based on the discussion above, the container sides would not yield spurious reflections if the scattered wavefield, caused by the topographic ground surface anomaly, were allowed adequate distance to attenuate. To give an order of magnitude sense, Bouckovalas and Papadimitriou [52] found that the free-field condition defined in terms of peak acceleration amplitude can be satisfied for distance from the crest anywhere between 2 to 8 times the slope height. The experimental design, however, entailed several conflicting constraints in this regard:

- The highest frequency (shortest wavelength) was bound by the actuator capacity $\left(f_{\max }=5.5 \mathrm{~Hz}\right.$, in prototype scale with centrifugal acceleration of $55 \mathrm{~g}, f_{\max }=300 \mathrm{~Hz}$ in model scale);

- In turn, because topographic effects manifest for slope dimensions comparable to the dominant excitation wavelength [2, 3, 11, 17, 53], the actuator's highest frequency also constrained the minimum slope dimension that would trigger topographic amplification effects;

- At the same time, the experiment was designed with dense sand $\left(G_{0}=108 \mathrm{MPa} ; D_{r}=100 \%\right)$ to prevent the slope stability failure during the test; thus, at $f_{\max }$, the shortest incident wavelength was $\lambda_{\min }=45 \mathrm{~m}$ (in prototype scale), whose effects would manifest for a slope of similar dimensions;

- The fixed container dimensions, however, could not accommodate the minimum distance between the toe and 
crest of the slope and the boundaries, that were required to avoid spurious reflections. [21]

Given these constraints, the slope-to-boundary distance was inevitably compromised during the test, creating the conditions for spurious reflections of the scattered wavefield. We next examined how strong these reflections were and to what extent, if any, they affected the recorded topographic amplification patterns using the finite element model shown in Figure 17. The material and slope geometry were identical to the centrifuge configuration described above, but the container box was in this case replaced by paraxial absorbing elements that minimize energy reflections [54]. In other words, the numerical model in Figure 17 is a slope on the surface of a Nevada sand layer overlying a practically rigid bedrock (here, the aluminum base plate). Boundary effects were revealed clearly when we examined the spatial distribution of peak (absolute maximum) acceleration on a model cross-section. Figure 18 compares the peak acceleration contours produced by the centrifuge experimental data, the full-container numerical simulations, and the extended free-field numerical simulations, for three different broadband ground motions of increasing intensity.

In the extended free-field simulations, the maximum amplification -while small- appeared in the vicinity of the crest, and the response gradually approached 1D conditions both behind the crest and away from the toe. The amount of amplification also appeared to increase with increasing motion intensity; specifically in the stronger of the three motions, the topographic amplification distribution strongly resembles the displacement field of a slope stability failure.

By contrast, the maximum amplification for both the centrifuge experimental data and the full-container numerical simulations, and regardless of the shaking intensity, was observed at the top left corner (see Figure 18). Considering that the interface between soil and container -the interface of a granular medium and a metal surface- has practically no cohesion, the top corner of the model responds during load reversal as a vertical $\left(90^{\circ}\right)$ slope, much more prone to localized amplification and slope stability failure than the $30^{\circ}$ slope; It is also possible that the pounding of the container rings may have accentuated the high shaking at the top left corner.

Note also that the peak acceleration contours of the centrifuge experimental data show a clear minimum at the midpoint of the model close to the base, and a radially increasing peak acceleration away from that point towards the bottom corners of the model. This effect, which did not appear in the fullcontainer simulations, is additional evidence of the rigid body rocking of the centrifuge container, which contributed to the increased level of acceleration near the container boundaries and at the surface compared to our extended "free-field" simulations.

We next estimated the effects of the lateral boundaries on the measured crest-to-free field spectral ratio. Figure 19 compares the spectral ratio predicted by the model shown in Figure 17, to the spectral ratio from the simulated centrifuge model test (Figure 2). Although the comparison of maximum acceleration contours shown in Figure 18 revealed significant lateral boundary effects, the crest-to-free field spectral ratios weren't significantly affected by lateral boundary reflections. This finding suggests that despite the prominent boundary effects in the physical model experiments, reflections attenuated rapidly and its influence on the response of the central part of the model where the topographic feature was located was small.

\subsection{Effects of the aluminum base plate}

We lastly examined the contribution of the base plate to the observed topographic amplification. For this purpose, we constructed a numerical model of a slope on the surface of a Nevada sand halfspace (refer to Figure 17). Here, both side boundaries and base plate were replaced with paraxial absorbing elements [54], and the incident motion was introduced in terms of incident stress. For more details on free-field boundary conditions for 2D site response problems, see Assimaki et al. [2, 53].

Comparison of the median surface-to-baseplate spectral ratios shown in Figure 20 for the sloped and flat ground model tests revealed that the crest to baseplate ratio of the former, and the flat ground to baseplate ratio from the latter, are almost identical in the frequency range $1-3 \mathrm{~Hz}$. In other words, in the frequency range of shaking $(1 \sim 2 \mathrm{~Hz})$, the two models have nearly identical, 1D site response. At frequencies higher than $3 \mathrm{~Hz}$, however, we observed a clear evidence of topographic effects, causing the differences in amplitude at $3.5 \mathrm{~Hz}$ and $6.5 \mathrm{~Hz}$, which manifest as topography amplification peaks in the ratio $A_{\text {Crest }}^{h} / A_{f f}^{h}$ shown in Figure 14.

We last compared the experimental topographic amplification factor (corrected using equation (5)) to the numerical amplification factor of the free-field halfspace model, defined by equation 8 .

$$
S R_{\text {Crest }}=\frac{A_{2 D}^{h}(\omega)}{A_{f f}^{h}(\omega)}
$$

where $A_{2 D}^{h}(\omega)$ and $A_{f f}^{h}(\omega)$ here describe the Fourier amplitude spectra of the response behind the crest and in the free-field, respectively, computed on the surface of the same model as shown in Figure 17, in contrast to equation (5), no correction was necessary in this case.

Figure 21 compares the two factors, and reveals fundamental differences between the numerical free-field halfspace model and the centrifuge experimental results: the former shows practically no topographic amplification in the entire frequency range $1-20 \mathrm{~Hz}$. Comparison between the free-field numerical models with and without baseplate (also in Figure 21), both free of boundary reflections from the centrifuge box, shows that the topographic amplification of the $30^{\circ}$ slope (at $3 \sim 4 \mathrm{~Hz}$ and $7 \sim 8 \mathrm{~Hz}$ ) most likely manifested as a consequence of energy reflections by the baseplate. In other words, the base plate in this 2D model trapped and amplified propagating waves -including the inclined reflected, converted and scattered from the surface slope- similarly to a 1D soil layer overlying bedrock. However, the traditional approach of normalizing the $2 \mathrm{D}$ response at the crest by the corresponding 1D surface motion does not remove 


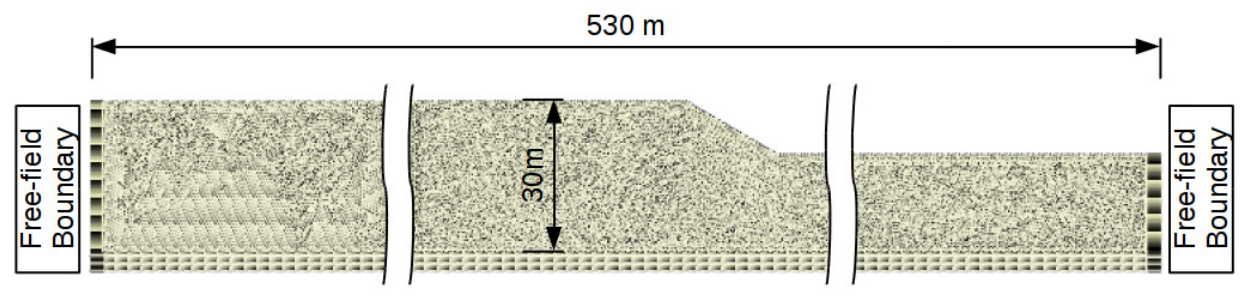

Figure 17: Finite element mesh with free-field boundary condition. The material and slope geometry are identical, but the model (soils and the aluminum base plate) is further extended to minimize the lateral boundary effect. Lateral boundaries are treated with paraxial absorbing elements and equivalent nodal forces.

the additional 2D amplification caused by the stiff layer; the topographic amplification with rigid layer is thus higher than the one resulting from the same geometry on a halfspace.

\section{Conclusions}

We presented a study where numerical simulations were used to understand and extend a study of centrifuge experiments on topographic amplification. First, we used the experimental data to validate the numerical models. Then, we identified limitations of centrifuge experiments for this class of problems, pertaining to conflicting constraints of frequency input, material stiffness, slope size, and container dimensions. Unavoidable trade-offs in the experimental design led to compromises in the distance of the scatterer (the slope) from the boundaries, which likely yielded spurious reflections. Our analysis also suggested that the rigid-body rocking motion of the centrifuge container likely increased the experimental topographic spectral ratios, contributing to the discrepancy between the simulated and observed spectral ratios.

Despite the compromise made in designing the experiment and the influence of the container rocking, we found that the lateral boundary effect had a small impact on the crest-to-free field spectral ratio. Our study also showed that the baseplate (or a stiff underlying layer in a free-field configuration) was instrumental in trapping and amplifying the non-1D scattered waves, which manifested as spectral ratio peaks at $3 \sim 4 \mathrm{~Hz}$ and $7 \sim 8 \mathrm{~Hz}$. In absence of the stiff baseplate, topographic amplification would have been much smaller.

Results of this study suggest that topographic effects are significantly affected by the underlying soil stratigraphy. Ground motion amplification on the crest in excess of 1D response was much more pronounced when we added a stiff layer several feet below, indicating that soil amplification is coupled with topographic effects. This finding, consistent with numerous previous studies [22, 25, 30, 55], is contrary to procedures of European design codes [56] and seismological models -where topographic effects are treated as path effects at best, and are thus superimposed (uncoupled) to 1D soil amplification factors. In this context, our study indicates that coupling of soil response and topographic effects is an important, poorly understood phenomenon that merits additional research, which should be accompanied by detailed geophysical investigations on elevated sites.
More importantly yet, our results demonstrate how combining centrifuge experimental data with numerical simulations can deepen our understanding of geotechnical earthquake engineering problems. Despite the limitations of the physical and numerical models presented in this study (e.g. PGA up to $0.6 \mathrm{~g}$, shear strain up to $0.1 \%$, and the effect of container boundary), they were each invaluable in providing an extensive and essential validation dataset and parametric search space correspondingly. The validated numerical models further analyzed enabled us to disentangle the interfering reflected waves recorded in the centrifuge, and to demonstrate that flexible container tests can be successfully used to understand the effects of topography in strong ground motion. Overall, the findings of this study clearly demonstrate that future studies will benefit from clear understanding and careful considerations of capabilities and limitations of the investigation methods and that the numerical modeling and the lab testing (or the field testing) methods should complement each other.

\section{Acknowledgements}

This material is based upon work supported by the National Science Foundation under Grants No. CMMI-0936543 and CMMI1132373 with title "NEESR-CR: Topographic Effects in Strong Ground Motion - From Physical and Numerical Modeling to Design", and Grant No. CMMI-0619078 with title "Topographic Amplification of Seismic Motion: Observations and Simulations in 3D". Any opinions, findings, and conclusions or recommendations expressed in this material are those of the author(s) and do not necessarily reflect the views of the National Science Foundation.

\section{References}

[1] J. Dafni, Experimental Investigation of the Topographic Modification of Earthquake Ground Motion, Ph.D. thesis, University of Washington, 2017.

[2] D. Assimaki, E. Kausel, G. Gazetas, Soil-dependent topographic effects: A case study from the 1999 Athens earthquake, Earthquake Spectra 21 (2005) 929-966.

[3] P.-Y. Bard, Diffracted waves and displacement field over two-dimensional elevated topographies, Geophysical Journal of the Royal Astronomical Society 72 (1982) 731-760.

[4] P.-Y. Bard, Local effects on strong ground motion: physical basis and estimation methods in view of microzoning studies, in: Proceedings of 

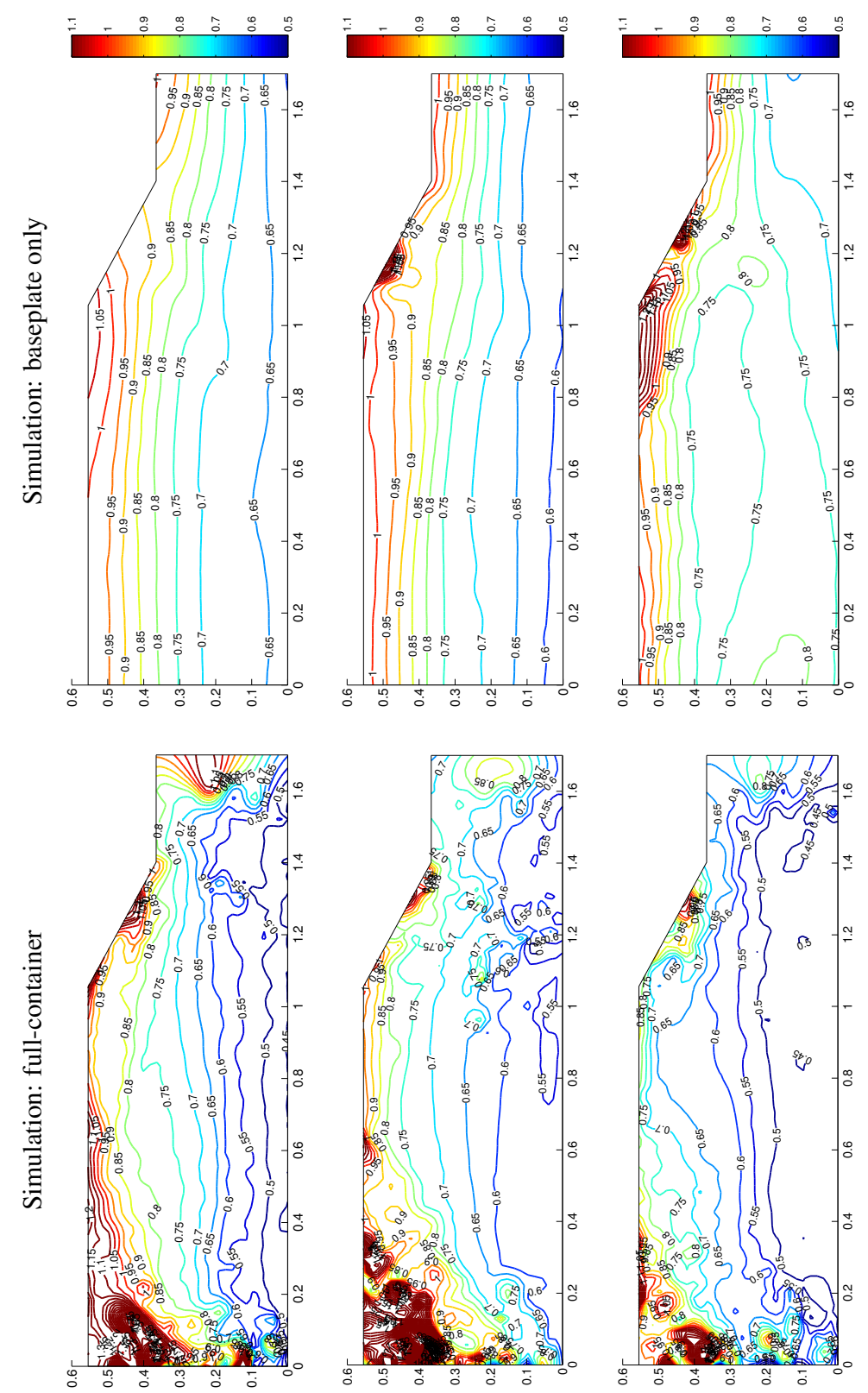

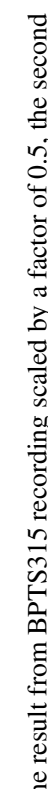
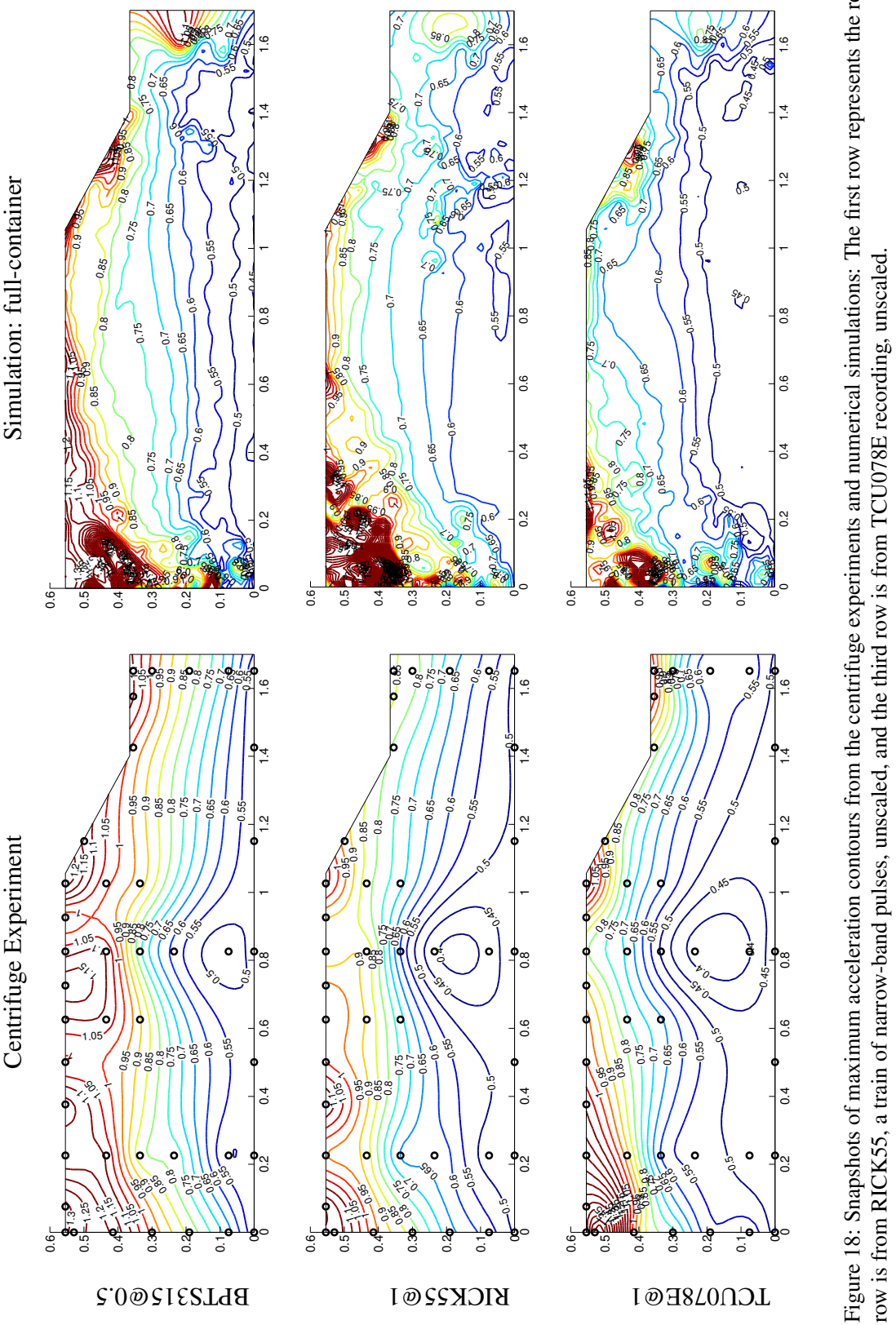


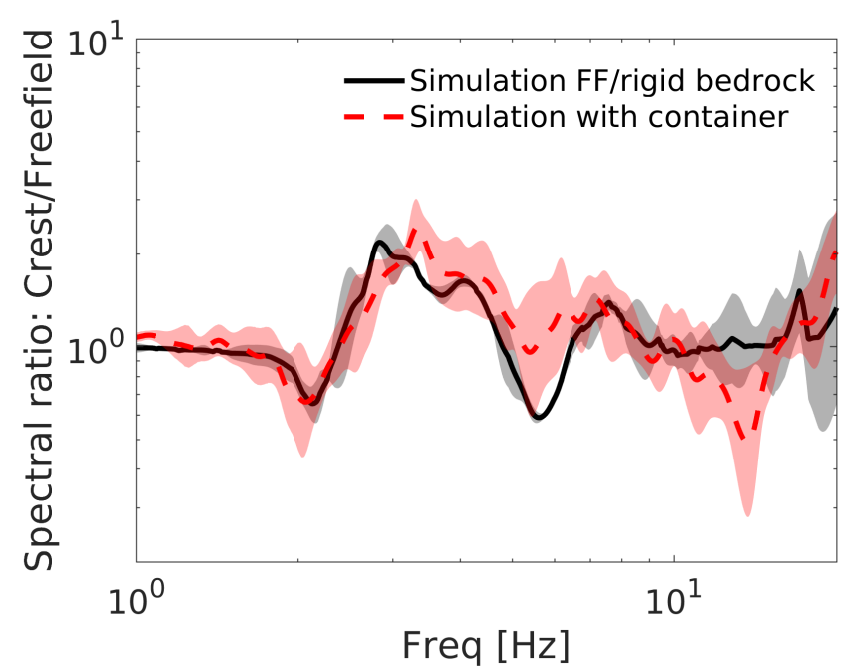

Figure 19: Comparison of median spectral ratios at the crest relative to the free field. The difference between two curves represent the effect of lateral boundary on the median crest-to-free field spectral ratio.

the Advanced Study Course on Seismotectonic and Microzonation Techniques in Earthquake Engineering, volume 4, Kefallinia, Greece, 1999. pp. 127-218.

[5] D. M. Boore, A note on the effect of simple topography on seismic SH waves, Bulletin of the seismological Society of America 62 (1972) 275284.

[6] M. Bouchon, Effect of topography on surface motion, Bulletin of the seismological Society of America 63 (1973) 615-632.

[7] M. Bouchon, J. Barker, Seismic response of a hill: The example of tarzana, california, Bulletin of the Seismological Society of America 86 (1996) 66-72.

[8] F. Nagashima, H. Kawase, S. Matsushima, F. J. Sanchez-Sesma, T. Hayakawa, T. Satoh, M. Oshima, Application of the H/V spectral ratios for earthquake ground motions and microtremors at K-NET sites in Tohoku region, Japan to delineate soil nonlinearity during the 2011 off the Pacific Coast of Tohoku earthquake, in: Proceedings of the International Symposium on Engineering Lessons Learned from the 2011 Great East Japan Earthquake, Tokyo, Japan, 2012

[9] E. Faccioli, Seismic amplification in the presence of geological and topographic irregularities, in: Proceedings of the $2^{\text {nd }}$ International Conference on Recent Advances in Geotechnical Earthquake Engineering and Soil Dynamics, volume 2, S. Louis, Missouri, 1991, pp. 1779-1797.

[10] W. Finn, Geotechnical engineering aspects of seismic microzonation, in: Proceedings of $4^{\text {th }}$ International Conference on Seismic Zonation, volume 1, EERI, Stanford, CA, 1991, pp. 199-250.

[11] L. Geli, P.-Y. Bard, B. Jullien, The effect of topography on earthquake ground motion: A review and new results, Bulletin of the Seismological Society of America 78 (1988) 42-63.

[12] S. Nechtschein, P.-Y. Bard, J.-C. Gariel, J.-P. Mèneroud, P. Dervin, M. Cushing, B. Gaubert, S. Vidal, A.-M. Duval, A topographic effect study in the nice region, in: Proceedings of the Fifth International Conference on Seismic Zonation, volume 2, Ouest Édition, Nice, France, 1995 pp. 1067-1074.

[13] F. Sanchez-Sesma, I. Herrera, J. Aviles, A boundary method for elastic wave diffraction: Application to scattering of $\mathrm{SH}$ waves by surface irregularities, Bulletin of the seismological Society of America 72 (1982) 473-490.

[14] W. D. Smith, The application of finite element analysis to body wave propagation problems, Geophysical Journal of the Royal Astronomical Society 42 (1975) 747-768.

[15] B. Tucker, J. King, D. Hatzfeld, I. Nersesov, Observations of hard rock site effects, Bulletin of the seismological Society of America 74 (1984) 121-136.

[16] M. Bouchon, C. A. Schultz, M. N. Toksöz, Effect of three-dimensional

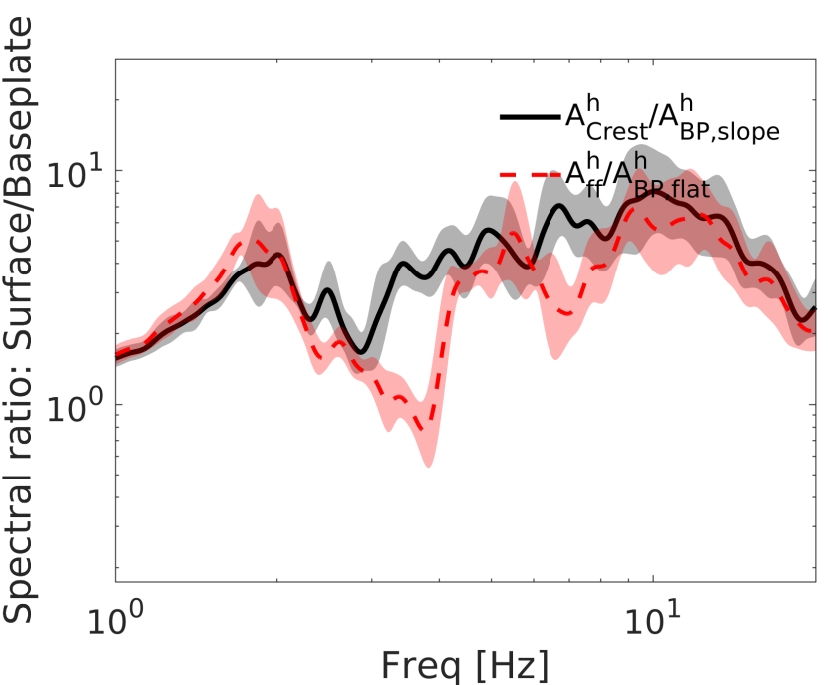

Figure 20: Comparison of the median surface to baseplate spectral ratio for flat and slope model. The peak at $2 \mathrm{~Hz}$ represents the fundamental mode of 1-D site response, which is almost unaffected by the topography effect.

topography on seismic motion, Journal of Geophysical Research 101 (1996) 5835-5846.

[17] S. A. Ashford, N. Sitar, J. Lysmer, N. Deng, Topographic effects on the seismic response of steep slopes, Bulletin of the seismological Society of America 87 (1997) 701-709.

[18] P.-Y. Bard, B. E. Tucker, Underground and ridge site effects: A comparison of observation and theory, Bulletin of the seismological Society of America 75 (1985) 905-922.

[19] F. J. Sanchez-Sesma, Diffraction of elastic waves by three-dimensional surface irregularities, Bulletin of the seismological Society of America 73 (1983) 1621-1636.

[20] Z. Zhenpeng, L. Baipo, Y. Yifan, Effect of three-dimensional topography on earthquake ground motion, in: Proceedings of the $7^{\text {th }}$ World Conference on Earthquake Engineering, volume 2, 1980, pp. 161-168.

[21] D. Assimaki, Topography effects in the 1999 Athens earthquake: Engineering issues in seismology, $\mathrm{PhD}$ Dissertation, Department of Civil and Environmental Engineering, Massachusetts Institute of Technology, 2004.

[22] R. Paolucci, E. Faccioli, F. Maggio, 3D Response analysis of an instrumented hill at Matsuzaki, Japan, by a spectral method, Journal of Seismology 3 (1999) 191-209.

[23] S. J. Lee, D. Komatitsch, B. S. Huang, J. Tromp, Effects of topography on seismic-wave propagation: An example from northern taiwan?, Bulletin of Seismological Society of America 99 (2009) 314-325

[24] E. Maufroy, V. M. Cruz-Atienza, S. Gaffeta, A robust method for assessing 3-d topographic site effects: A case study at the 1sbb underground laboratory, france, Earthquake Spectra 28 (2012) 1097-1115.

[25] J. Burjánek, B. Edwards, D. Fäh, Empirical evidence of local seismic effects at sites with pronounced topography: A systematic approach, Geophysical Journal International 197 (2014) 608-619.

[26] J. Burjánek, D. Fäh, M. Pischiutta, A. Rovelli, G. Calderoni, P.-y. Bard, Site effects at sites with pronounced topography : overview \& recommendations Site effects at sites with pronounced topography : overview \& recommendations, Technical Report, 2014. doi 10.3929/ ethz-a-010222426.Site

[27] M. Pischiutta, G. Cultrera, A. Caserta, L. Luzi, A. Rovelli, Topographic effects on the hill of Nocera Umbra, central Italy, Geophysical Journal International 182 (2010) 977-987.

[28] M. Massa, S. Barani, S. Lovati, Overview of topographic effects based on experimental observations: Meaning, causes and possible interpretations, Geophysical Journal International 197 (2014) 1537-1550.

[29] M. R. Gallipoli, M. Bianca, M. Mucciarelli, S. Parolai, M. Picozzi, Topographic versus stratigraphic amplification: Mismatch between code provisions and observations during the L'Aquila (Italy, 2009) sequence, Bul- 

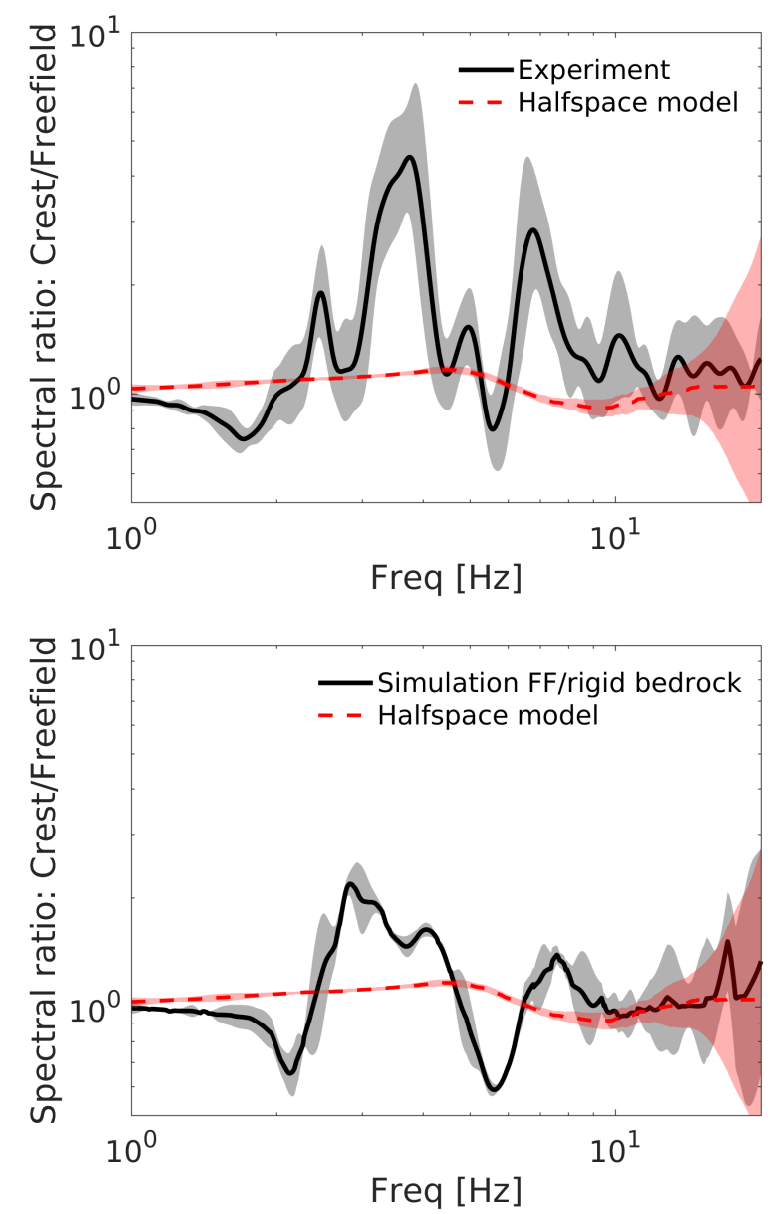

Figure 21: Median spectral ratios at the crest relative to the free field: container boundary vs free-field boundary. Horizontal accelerations recorded at the crest with halfspace model show negligible topographic effects, indicating the topography effect observed during experiments are highly attributed to the presence of a nearly rigid baseplate.

letin of Earthquake Engineering 11 (2013) 1325-1336.

[30] V. Graizer, Low-velocity zone and topography as a source of site amplification effect on tarzana hill, california, Soil Dynamics and Earthquake Engineering 29 (2009) 324-332.

[31] D. Assimaki, S. Jeong, Groundmotion observations at hotel montana during the m 7.0 2010 haiti earthquake: Topography or soil amplification?, Bulletin of Seismological Society of America 103 (2013) 2577-2590.

[32] S. Hartzell, M. Meremonte, L. Ramírez-Guzmán, D. McNamara, Ground motion in the presence of complex topography: Earthquake and ambient noise sources, Bull. Seismol. Soc. Am. 104 (2014) 451-466.

[33] S. Hartzell, L. RamírezGuzmán, M. Meremonte, A. Leeds, Ground Motion in the Presence of Complex Topography II: Earthquake Sources and 3D Simulations, Bull. Seismol. Soc. Am. 107 (2016) 344-358.

[34] J. Dafni, J. Wartman, Experimental Investigation of Topographic Effects Using a Centrifuge Model: Test 1, NHERI: A Natural Hazards Engineering Research Infrastructure (distributor), 2013. URL: https://www.designsafe-ci.org/data/browser/ public/nees.public/NEES-2010-0977.groups/Experiment-3 doi $10.4231 /$ D3T14TP1T

[35] B. L. Kutter, I. Idriss, T. Khonke, J. Lakeland, X. Li, W. Sluis, X. Zeng, R. C. Tauscher, Y. Goto, I. Kubodera, Design of a large earthquake simulator at uc davis, in: Centrifuge 94, International Society of Soil Mechanics and Foundation Engineering, Balkema, 1994, pp. 169-175.

[36] J. Prevost, DYNAFLOW: A nonlinear transient finite element analysis program, Technical Report, Dept. of Civil Eng. and Op. Research, Princeton University, 1995.
[37] J. Snowdon, Vibration and Shock in Damped Mechanical Systems, Wiley, 1968.

[38] J. Prevost, A simple plasticity theory for frictional cohesionless soils, Soil Dynamics and Earthquake Engineering 4 (1985) 9-17.

[39] R. L. Kondner, Hyperbolic stress-strain response: Cohesive soils, Journal of the Soil Mechanics and Foundations Division, ASCE 89 (1963) 115143.

[40] H. Hayashi, M. Honda, T. Yamada, F. Tatsuoka, Modeling of nonlinear stress strain relations of sands for dynamic response analysis, in: Tenth World Conference on Earthquake Engineering, Balkema, 1994.

[41] G. Masing, Eigenspannungen und verfestigung beim messing, in: Proc. Second Int. Congr. Appl. Mech., Zurich, Switzerland, 1926, pp. 332-335.

[42] D. Stevens, B.-I. KIM, D. Wilson, B. Kutter, Comprehensive investigation of nonlinear site response - Centrifuge data report for DKS02, Center for Geotechnical Modeling UCD/CGMDR-99/02, University of California at Davis, 1999.

[43] M. B. Darendeli, Development of a new family of normalized modulus reduction and material damping curves, Ph.D. thesis, University of Texas at Austin, 2001.

[44] H. M. Hilber, T. J. Hughes, R. L. Taylor, Improved numerical dissipation for time integration algorithms in structural dynamics, Earthquake Engineering \& Structural Dynamics 5 (1977) 283-292.

[45] T. J. Hughes, The Finite Element Method: Linear Static and Dynamic Finite Element Analysis, Prentice Hall, 1987.

[46] A. Elgamal, Z. Yang, T. Lai, B. L. Kutter, D. W. Wilson, Dynamic response of saturated dense sand in laminated centrifuge container, Journal of Geotechnical and Geoenvironmental Engineering 131 (2005) 598-609.

[47] P. M. Byrne, S.-S. Park, M. Beaty, M. Sharp, L. Gonzalez, T. Abdoun, Numerical modeling of liquefaction and comparison with centrifuge tests, Canadian Geotechnical Journal 41 (2004) 193-211.

[48] M. Kristeková, J. Kristek, P. Moczo, Time-frequency misfit and goodness-of-fit criteria for quantitative comparison of time signals, Geophysical Journal International 178 (2009) 813-825.

[49] M. Beyreuther, R. Barsch, L. Krischer, T. Megies, Y. Behr, J. Wassermann, ObsPy: A Python Toolbox for Seismology, Seismol. Res. Lett. 81 (2010) 530-533.

[50] R. V. Whitman, P. C. Lambe, Effect of boundary conditions upon centrifuge experiments using ground motion simulation, Geotechnical Testing Journal 9 (1986) 61-71.

[51] A. Schofield, X. Zeng, Design and performance of an Equivalent-ShearBeam (ESB) container for earthquake centrifuge modelling, Department of Engineering Report CUED/D-SOILS/TR245, University of Cambridge, 1992.

[52] G. D. Bouckovalas, A. G. Papadimitriou, Numerical evaluation of slope topography effects on seismic ground motion, Soil Dynamics and Earthquake Engineering 25 (2005) 547-558.

[53] D. Assimaki, G. Gazetas, E. Kausel, Effects of local soil conditions on the topographic aggravation of seismic motion: Parametric investigation and recorded field evidence from the 1999 athens earthquake, Bulletin of the seismological Society of America 95 (2005) 1059-1089.

[54] R. W. Clayton, B. Engquist, Absorbing boundary conditions for acoustic and elastic wave equations, Bulletin of the Seismological Society of America 67 (1977) 1529-1540.

[55] R. Tripe, S. Kontoe, T. Wong, Slope topography effects on ground motion in the presence of deep soil layers, Soil Dynamics and Earthquake Engineering 50 (2013) $72-84$.

[56] EC8, Design provisions for earthquake resistance of structures, part 11: General rules-seismic actions and general requirements for structures, prEN 1998-5, European Committee for Standardization, Brussels, 2000. 